OPEN ACCESS

Edited by:

Xihui Shen

Northwest A\&F University, China

Reviewed by:

Qiyao Wang,

East China University of Science and

Technology, China

Jeanette Elaine Bröms,

Umeå University, Sweden

*Correspondence:

Biao Kan

Kanbiao@icdc.cn

Weili Liang

liangweili@icdc.cn

Specialty section:

This article was submitted to

Infectious Diseases,

a section of the journal

Frontiers in Microbiology

Received: 28 December 2016 Accepted: 14 March 2017 Published: 30 March 2017

Citation:

Huang Y, Du P, Zhao M, Liu W, Du Y,

Diao B, Li J, Kan B and Liang W

(2017) Functional Characterization and Conditional Regulation of the Type VI Secretion System in Vibrio fluvialis.

Front. Microbiol. 8:528.

doi: 10.3389/fmicb.2017.00528

\section{Functional Characterization and Conditional Regulation of the Type VI Secretion System in Vibrio fluvialis}

\author{
Yuanming Huang ${ }^{1,2}$, Pengcheng Du ${ }^{3}$, Meng Zhao ${ }^{1}$, Wei Liu ${ }^{1}$, Yu Du ${ }^{1}$, Baowei Diao ${ }^{1}$, \\ Jie $\mathrm{Li}^{1}$, Biao Kan ${ }^{1,2 *}$ and Weili Liang ${ }^{1,2 *}$
}

${ }^{1}$ State Key Laboratory of Infectious Disease Prevention and Control, National Institute for Communicable Disease Control and Prevention, Chinese Center for Disease Control and Prevention, Beijing, China, ${ }^{2}$ Collaborative Innovation Centre for Diagnosis and Treatment of Infectious Diseases, Hangzhou, China, ${ }^{3}$ Beijing Key Laboratory of Emerging Infectious Diseases, Institute of Infectious Diseases, Beijing Ditan Hospital, Capital Medical University, Beijing, China

Vibrio fluvialis is an emerging foodborne pathogen of increasing public health concern. The mechanism(s) that contribute to the bacterial survival and disease are still poorly understood. In other bacterial species, type VI secretion systems (T6SSs) are known to contribute to bacterial pathogenicity by exerting toxic effects on host cells or competing bacterial species. In this study, we characterized the genetic organization and prevalence of two T6SS gene clusters (VfIT6SS1 and VfIT6SS2) in V. fluvialis. VfIT6SS2 harbors three "orphan" hcp-vgrG modules and was more prevalent than VfIT6SS1 in our isolates. We showed that VfIT6SS2 is functionally active under low $\left(25^{\circ} \mathrm{C}\right)$ and warm $\left(30^{\circ} \mathrm{C}\right)$ temperatures by detecting the secretion of a T6SS substrate, Hcp. This finding suggests that VfIT6SS2 may play an important role in the survival of the bacterium in the aquatic environment. The secretion of Hcp is growth phase-dependent and occurs in a narrow range of the growth phase $\left(\mathrm{OD}_{600}\right.$ from 1.0 to 2.0). Osmolarity also regulates the function of VfIT6SS2, as evidenced by our finding that increasing salinity (from 170 to $855 \mathrm{mM}$ of $\mathrm{NaCl}$ ) and exposure to high osmolarity $\mathrm{KCl}$, sucrose, trehalose, or mannitol (equivalent to $340 \mathrm{mM}$ of $\mathrm{NaCl}$ ) induced significant secretion of $\mathrm{Hcp}$ under growth at $30^{\circ} \mathrm{C}$. Furthermore, we found that although VfIT6SS2 was inactive at a higher temperature $\left(37^{\circ} \mathrm{C}\right)$, it became activated at this temperature if higher salinity conditions were present (from 513 to $855 \mathrm{mM}$ of $\mathrm{NaCl}$ ), indicating that it may be able to function under certain conditions in the infected host. Finally, we showed that the functional expression of VflT6SS2 is associated with anti-bacterial activity. This activity is Hcp-dependent and requires vasH, a transcriptional regulator of T6SS. In sum, our study demonstrates that VfIT6SS2 provides $V$. fluvialis with an enhanced competitive fitness in the marine environment, and its activity is regulated by environmental signals, such as temperature and osmolarity.

Keywords: Type VI secretion system, Hcp, VasH, T6SS, bacterial killing, Vibrio fluvialis

Abbreviations: T6SS, type VI secretion system; Hcp, hemolysin-coregulated protein; VgrG, valine-glycine repeat protein G; LB, Luria-Bertani broth; WT, wild-type; OD600, optical density at $600 \mathrm{~nm}$; PCR, polymerase chain reaction; CFU, colony forming units; ORF, open reading frame; VFH, hemolysin of $V$. fluvialis. 


\section{INTRODUCTION}

$V$. fluvialis, a halophilic Gram-negative bacterium, is an emerging foodborne pathogen of increasing public health concern. It was originally isolated in 1975 from a patient suffering from severe diarrhea and was called "group F Vibrio" and "EF-6 Vibrio" by different research groups. The name $V$. fluvialis was proposed later by Lee et al. (1981). Since its discovery, the microbe has been implicated in both outbreaks and sporadic cases of diarrhea (Huq et al., 1980; Bellet et al., 1989; Klontz and Desenclos, 1990; Srinivasan et al., 2006; Bhattacharjee et al., 2010; Chowdhury et al., 2012), as well as various extra-intestinal infections (Huang and Hsu, 2005; Ratnaraja et al., 2005; Lai et al., 2006; Liu et al., 2011). Infection by $V$. fluvialis is most common in infants, children, and young adults. There has recently been an increase in the isolation rate from diarrheal patients, and multidrug-resistant clinical isolates of $V$. fluvialis have been reported (Ahmed et al., 2005; Srinivasan et al., 2006; Chowdhury et al., 2011, 2012). The clinical symptoms of $V$. fluvialis gastroenteritis are similar to those of Vibrio cholerae, except for the occurrence of bloody stool in $V$. fluvialis infection, which is a notable difference from cholera (Huq et al., 1980; Allton et al., 2006).

Despite increasing public health concern about $V$. fluvialis, knowledge about its microbiological characteristics, virulence factors, environmental fitness, and the epidemiology of infection is still limited (Igbinosa and Okoh, 2010; Liang et al., 2013). Many pathogenic factors, such as proteases, cytolysins, Chinese hamster_ovary $(\mathrm{CHO})$ cell elongation factor, $\mathrm{CHO}$ cell-killing factor, enterotoxin-like substance, lipase, and hemolysin have been isolated and characterized, but their precise roles in the clinical manifestations and the pathogenicity of the bacterium remain to be explored (Lockwood et al., 1982; Chikahira and Hamada, 1988; Miyoshi et al., 2002; Kothary et al., 2003; Cabrera Rodríguez et al., 2005; Igbinosa and Okoh, 2010). Of the many virulence factors, hemolysin was thought to be of greatest importance. It was reported that hemolysin of $V$. fluvialis (VFH) formed larger pores in the erythrocyte membrane than other Vibrio hemolysins, including $V$. cholerae and Vibrio vulnificus (Han et al., 2002; Igbinosa and Okoh, 2010). Our previous study demonstrated that VFH contributes to the pathogenicity of $V$. fluvialis by inducing IL- $1 \beta$ secretion through the activation of the NLRP3 inflammasome (Song et al., 2015). We also showed that quorum sensing in $V$. fluvialis positively regulates the production of hemolysin and extracellular protease and affects the cytotoxic activity against epithelial tissue cultures (Wang et al., 2013).

The type VI secretion system (T6SS) is a newly discovered contact-dependent protein secretion system. Although the existence of T6SS had been postulated for more than a decade before, the T6SS was first functionally identified in O37 $\mathrm{V}$. cholerae and Pseudomonas aeruginosa by the Mekalanos group in 2006 (Mougous et al., 2006; Pukatzki et al., 2006). In V. cholerae, it was demonstrated that the extracellular export of hemolysincoregulated protein (Hcp) and three related valine-glycine repeat protein $\mathrm{G}$ (VgrG) proteins were required for the cytotoxicity of the organism in amoebae and macrophages (Pukatzki et al., 2006). In P. aeruginosa, HSI-I T6SS mediated the export of Hcp1, and evoked an immune response in cystic fibrosis (Mougous et al., 2006). Subsequent to these two landmark publications, numerous follow-up studies have demonstrated that T6SS is involved in the virulence and environmental competitive fitness of various bacterial species, such as Burkholderia species (Schell et al., 2007; Shalom et al., 2007), Edwardsiella tarda (Zheng and Leung, 2007), Agrobacterium tumefaciens (Wu et al., 2012), Vibrio parahaemolyticus (Yu et al., 2012), Aeromonas hydrophila (Suarez et al., 2008), Citrobacter freundii (Liu et al., 2015), Vibrio alginolyticus (Sheng et al., 2012; Salomon et al., 2015), and Burkholderia cenocepacia (Aubert et al., 2016). A genomelevel survey revealed that more than $25 \%$ of genome-sequenced Gram-negative species contain T6SS gene clusters (Bingle et al., 2008). Although there are variations in their genetic contents and organization, 13 core T6SS genes have been recognized (Boyer et al., 2009). The T6SS secretion apparatus is structurally and functionally analogous to a contractile T4 bacteriophage tail, but has a reversed orientation (Leiman et al., 2009). A working model of the T6SS proposes that an intracellular Hcp nanotube, with a spike-like structure composed of a trimer of VgrG and PAAR (proline, alanine, alanine, arginine) motif-containing proteins at its top, is pushed through the envelope of the predator cell and into an adjacent target cell following the secretion of Hcp, VgrG, and other effectors (Shneider et al., 2013; Ho et al., 2014). This ejection process is powered by the contraction of the dynamic VipA/VipB sheath surrounding the Hcp nanotube (Basler et al., 2012). The T6SS is tightly regulated and has crosstalk with other regulatory systems, including the competence regulon, quorum sensing, and stress response (Ishikawa et al., 2009; Weber et al., 2009; Borgeaud et al., 2015).

We recently reported the draft genome of a clinically-isolated strain of $V$. fluvialis, 85003, and the results of a sequence analysis which revealed the presence of homologs of T6SS (Lu et al., 2014). However, questions about the genetic organization, functions, and regulation of these homologs remain unanswered. In this study, we aimed to further characterize the genetic contents and organization, functions, and the environmental conditions that control the expression of $V$. fluvialis T6SS. We found that there are two T6SSs in strain 85003, VflT6SS2 ( $V$. fluvialis T6SS2), and VflT6SS1 ( $V$. fluvialis T6SS1), with the former more prevalent than the latter in different $V$. fluvialis isolates. The genetic contents and organization of VflT6SS2 are highly homologous to the T6SS of $V$. cholerae, but contains three differently located "orphan" hcp-vgrG modules in distinct locations. Detection of Hcp expression and secretion revealed that VflT6SS2 is functionally activated under low $\left(25^{\circ} \mathrm{C}\right)$ and warm $\left(30^{\circ} \mathrm{C}\right)$ temperatures, but is inactive at the higher temperature $\left(37^{\circ} \mathrm{C}\right)$. The secretion of Hcp is growth phase-dependent and only occurs at certain parts of the growth phase at $30^{\circ} \mathrm{C}$. Osmolarity positively regulates the VflT6SS2 activity. Increasing the salinity (from 170 to $855 \mathrm{mM}$ of $\mathrm{NaCl}$ ) and exposure to high osmolarity $\mathrm{KCl}$, sucrose, trehalose, or mannitol (equivalent to $340 \mathrm{mM} \mathrm{NaCl}$ ) induced the secretion of Hcp. Furthermore, VflT6SS2 could also be activated at $37^{\circ} \mathrm{C}$ if higher salinity conditions were present (513 $855 \mathrm{mM} \mathrm{NaCl}$ ). In addition, a bacterial killing assay demonstrated that functional expression of VflT6SS2 has antibacterial activity which is dependent on Hcp and requires the T6SS transcriptional regulator, VasH. 


\section{MATERIALS AND METHODS}

\section{Bacterial Strains, Culture Conditions, and Plasmids}

The bacterial strains and plasmids used in this study are listed in Table 1. The wild-type (WT) V. fluvialis 85003 and its derivative, as well as other $V$. fluvialis environmental and clinical isolates, were routinely grown in Luria-Bertani (LB) broth $(\mathrm{pH} 7.4)$ containing $170 \mathrm{mM} \mathrm{NaCl}$ at $30^{\circ} \mathrm{C}$ unless specifically indicated. E. coli SM10 $\lambda$ pir and Rosetta (DE3) were routinely cultured at $37^{\circ} \mathrm{C}$. Culture media were supplemented with ampicillin (Amp, $100 \mu \mathrm{g} / \mathrm{ml}$ ), streptomycin (Sm, $100 \mu \mathrm{g} / \mathrm{ml}$ ), rifampicin (Rfp, 50 $\mu \mathrm{g} / \mathrm{ml})$, kanamycin $(\mathrm{Km}, 50 \mu \mathrm{g} / \mathrm{ml})$, tetracycline $(\mathrm{Tc}, 10 \mu \mathrm{g} / \mathrm{ml}$ for E. coli, $2.5 \mu \mathrm{g} / \mathrm{ml}$ for $V$. fluvialis), chloramphenicol $(\mathrm{Cm}$, $10 \mu \mathrm{g} / \mathrm{ml}$ ), or isopropyl- $\beta$-D-thiogalactopyranoside (IPTG), as required.

\section{Determination of hcp-vgrG Alleles and Prevalence of T6SSs}

To determine the sequence of the 3' end of VflT6SS2, primers vfu-vasL-up2153177 and vfu-rbsD-dn2158250 were designed according to the corresponding sequence of Vibrio furnissii NCTC11218. Similarly, primer pairs vfuA01956-up/vfuA01959$\mathrm{dn}$ and vfuB01009-up/vfuB01011-dn were designed to amplify the $h c p$-vgrG alleles in 85003. The polymerase chain reaction
(PCR) was performed with TranStart FastPfu Fly DNA polymerase (Transgen Biotech, China) with 85003 genomic DNA used as a template and the products were used for commercial sequencing.

To determine whether T6SS genetic loci are prevalent in isolates of $V$. fluvialis, PCR was performed with waterboiled templates of isolates to detect the presence of multiple chromosomal fragments specific for each T6SS gene cluster. For VflT6SS1, fragments covering tssC1 (impC)-impD, impDtssF1 (impG), tssF1 (impG)-tssG1 (impH), tssH1, tssH1 (clpB)-tagH1 (impI), tagH1 (impI)-tssK1 (impJ), and tssL1 (impK)-tssM1 (impL) were respectively amplified by using primer pairs impC/impD-F and impC/impD-R, impD/impG$\mathrm{F}$, and impD/impG-R, impG/impH-F and impG/impH-R, clpB-F/clpB-R, clpB/impI-F and clpB/impI-R, lip/impJ-F, and lip/impJ-R, and impK/impL-F and impK/impL-R. For VflT6SS2, fragments covering tss $C 2$ (vipB)-tssF2 (vas $A$ ), tss $G 2$ (vasB)tagH2 (vasC), tssJ2 (vasD)-tssK2 (vasE), tssL2 (vasF)-tssH2 (vasG), vasH-vasI, vasI-tssA2 (vasJ), and tssM2 (vasK)-vasL were separately amplified with primer pairs vipB/vasA-F and vipB/vasA-R, vasB/vasC-F and vasB/vasC-R, vasD/vasE-F, and vasD/vasE- $\mathrm{R}$, vasF/vasG-F and vasF/vasG- $\mathrm{R}$, vasH/vasI-F and vasH/vasI $-\mathrm{R}$, vasI/vasJ-F, and vasI/vasJ-R, and vasK/vasL-F and vasK/vasL-R. The oligonucleotide primers used are listed in Table 2.

TABLE 1 | Strains and plasmids used in this study.

\begin{tabular}{|c|c|c|}
\hline Strain/plasmid & Characteristics & References/sources \\
\hline \multicolumn{3}{|l|}{ E. coli } \\
\hline SM10גpir & thi thr leu tonA lacY supE recA::RP4-2Tc::Mu ( $\lambda$ pirR6K), $\mathrm{Km}^{\mathrm{R}}$ & $\begin{array}{l}\text { Mekalanos Laboratory (Harvard Medical } \\
\text { School) }\end{array}$ \\
\hline Rosetta(DE3) & $\mathrm{F}^{-}$ompT hsdS $\mathrm{B}_{\mathrm{B}}\left(\mathrm{r}_{\mathrm{B}}^{-} \mathrm{m}_{\mathrm{B}}^{-}\right) \mathrm{gal} d c m(\mathrm{DE} 3) \mathrm{pRARE} \mathrm{E}^{2}, \mathrm{Cm}^{\mathrm{R}}$ & Laboratory stock \\
\hline MG1655 & $\mathrm{K}-12 \mathrm{~F}^{-} \lambda^{-} i / v \mathrm{G}^{-} \mathrm{rfb}-50 \mathrm{rph}-1, \mathrm{Rfp}^{\mathrm{R}}$ & Laboratory stock \\
\hline VF54 & V. fluvialis, wild type, environmental isolate & This study \\
\hline VF42 & V. fluvialis, wild type, clinical isolate & This study \\
\hline ClCC21612 & V. fluvialis, wild type, clinical isolate & Liang et al., 2013 \\
\hline$\Delta$ vasH & 85003, $\Delta$ vasH & This study \\
\hline$\Delta t s s D 2 a$ & 85003, $\Delta t s s D 2 \_a$ & This study \\
\hline pCVD442 & Suicide vector containing R6K ori, $\operatorname{sacB}, \mathrm{Amp}^{\mathrm{R}}$ & Laboratory stock \\
\hline pWM91 & Suicide vector containing R6K ori, $\operatorname{sac} B$, lacZ $\alpha ; A m p^{R}$ & Laboratory stock \\
\hline pSRKTc & Broad-host-range vector containing lac promoter, lac $/ q$, lac $Z \alpha$, Tet $^{R}$ & Khan et al., 2008 \\
\hline pET30a $(+)$ & expression vector containing pBR322 ori, f1 ori, lacl; Kan ${ }^{R}$ & Novagen \\
\hline pCVD-VFAvasH & $1.79 \mathrm{~kb}$ Sall-Sacl $\Delta$ vasH fragment of $V$. fluvialis in pCVD442 & This study \\
\hline pCVD- $\Delta t s s D / 2 \_c$ & $1.69 \mathrm{~kb}$ Sall-Sacl $\Delta t s s D_{-} c-t s s / 2 \_c$ fragment of $V$. fluvialis in pCVD442 & This study \\
\hline pWM- $\Delta t s s D 2 \_a$ & $1.74 \mathrm{~kb}$ BamHI-Smal $\Delta t s s D 2 \_a$ fragment of $V$. fluvialis in pWM91 & This study \\
\hline pWM- $\Delta t s s D 2 \_b$ & $1.72 \mathrm{~kb}$ Notl-Smal $\Delta t s s D 2 \_b$ fragment of $V$. fluvialis in pWM91 & This study \\
\hline pSRvasH & $1.596 \mathrm{~kb}$ vasH ORF of $V$. fluvialis in pSRKTc & This study \\
\hline pET30a-hcp & $513 \mathrm{bp}$ Ncol-Xhol fragment containing the coding sequence of $V$. fluvialis $h c p$ in pET30a(+) & This study \\
\hline
\end{tabular}




\section{Construction of Mutants and Complementation Plasmids}

The in-frame deletion mutants $\Delta v a s H, \Delta t s s D 2 \mathrm{a}, \Delta t s s D 2 \mathrm{~b}$, and $\Delta t s s D I 2 c$ were constructed by allelic exchange using the clinical strain 85003 as a WT precursor as described previously (Wu et al., 2015). We used $\Delta t s s D 2 \mathrm{a}$ as a precursor to construct the double mutant, $\Delta t s s D 2 \mathrm{ab}$. Briefly, the chromosomal fragments containing upstream and downstream nucleotide sequences of target genes were respectively amplified using the corresponding primers listed in Table 2 and then were stitched together by overlapping PCR. The $1.79 \mathrm{~kb} \Delta v a s H$ and $1.69 \mathrm{~kb} \Delta t s s D \_c$ tssI2_c fragments were individually cloned at SalI-SacI sites in a pCVD442 suicide plasmid to yield pCVD-VF $\Delta$ vasH and pCVD- $\Delta t s s D I 2 \_c$. The $1.74 \mathrm{~kb} \Delta t s s D 2 \_a$ and $1.72 \mathrm{~kb} \Delta t s s D 2 \_b$ fragments were cloned at BamHI-SmaI sites and NotI-SmaI sites in the suicide plasmid pWM91 to yield pWM- $\Delta t s s D 2 \_a$ and pWM- $\Delta t s s D 2 \_b$, respectively. The resulting recombinant suicide plasmids were conjugated into $V$. fluvialis from E. coli SM10 $\lambda$ pir and transconjugants were selected on LB media containing Amp and $\mathrm{Sm}$. The transconjugants were counter-selected by growing them on media containing $15 \%$ sucrose. Sucrose-resistant colonies were tested for Amp sensitivity, and target deletion was identified by PCR and confirmed by DNA sequencing.

The vasH expression plasmid was constructed for complementation test. Primers vasH-For-HindIII and vasH-RevNdeI were used to amplify the ORF of vasH with PrimeSTAR ${ }^{\circledR}$ HS DNA Polymerase (TaKaRa, Dalian, China) using WT 85003 genomic DNA as a template. The 1,596 bp PCR product with blunt ends was digested with $\mathrm{NdeI}$ and cloned at NdeI and SmaI sites in pSRKTc to generate pSRvasH, which expresses vasH from the lac promoter with the induction of IPTG. The pSRvasH

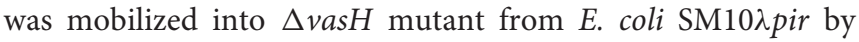
conjugation, and the expression vector pSRKTc without an insert was used as a negative control.

\section{Anti-Hcp Polyclonal Antiserum Preparation}

An anti-Hcp polyclonal antibody was raised in rabbits using the following method: First, the $h c p$ gene was amplified by PCR using primer pair VF-hcp-up-NcoI/VF-hcp-dn-XhoI and was cloned into pET30a $(+)$ using $N c o I$ and XhoI restriction enzyme sites. His-tagged Hcp expression was induced by IPTG in E. coli Rosetta (DE3) host cells and was purified by NiIDA affinity chromatography (Novagen) under native conditions according to the manufacturer's instructions. The purified Hcp protein was used for immunization of rabbits by Wuhan Abzome Biotechnology, China. The polyclonal antiserum was purified with an antigen affinity purification procedure before use.

\section{Analysis of T6SS Protein Production and Secretion}

Overnight cultures of bacterial strains were diluted to 1:100 in $20 \mathrm{~mL}$ of fresh $\mathrm{LB}$ containing $170 \mathrm{mM} \mathrm{NaCl}$ with shaking, and were incubated to an optical density at $600 \mathrm{~nm}\left(\mathrm{OD}_{600}\right)$ of 1.5. In the vasH complementation test, overnight cultures of $\Delta$ vas $H$ mutants containing plasmid pSRvasH $(\Delta v a s H / p S R v a s H)$
TABLE 2 | Primers used in this study.

\begin{tabular}{|c|c|}
\hline Primer & Oligonucleotide sequence $\left(5^{\prime}-3^{\prime}\right)$ \\
\hline vfu-vasL-up2153177 & CGTTGGAAGATITGCTGTG \\
\hline vfu-rbsD-dn2158250 & GTGAGGGCTAAATCAATACG \\
\hline vfuA01956-up & CCGCCATTTCGCTTACG \\
\hline vfuA01959-dn & GCTTGATCCCAACCGCAG \\
\hline vfu_B01009-up & CATTCAGGAAGATCCAGT \\
\hline vfu_B01011-dn & GCTGCCAATCAGTTCCTA \\
\hline impC/impD-F & AGCAATTTGAAGACGCACCT \\
\hline impC/impD-R & GGCGAGAAAAATCAGGATGA \\
\hline impD/impG-F & CAATCGCATCAACTGGTTTG \\
\hline impD/impG-R & GCAACACCGCAATCACATAC \\
\hline impG/impH-F & TGGACGAAGTCAACGAAGTG \\
\hline impG/impH-R & ACACAGAAGGCGCTTGAGTT \\
\hline clpB-F & TGTCCAAAGAAACGCATCTG \\
\hline clpB-R & GAACACCATGGGCTCTTCAT \\
\hline clpB/impl-F & ACGTCCGGAAAATGACGAT \\
\hline clpB/impl-R & TAAGGTGTAGGCCCCAATCA \\
\hline lip/impJ-F & TTCTGGCTGGGGTAACAAAG \\
\hline lip/impJ-R & AGTTCAGCGGAAAAGCTCAC \\
\hline impK/impL-F & AGCTCTACGCTCTGGGTTGA \\
\hline impK/impL-R & ACTCGTCGTGCTGCTTGAG \\
\hline vipB/vasA-F & CACCGATITGGCAGTGCTT \\
\hline vipB/vasA-R & GCTGCAAAGCAAGATCCCAA \\
\hline vasB/vasC-F & AAAACgCACCATCAATCgAgACA \\
\hline vasB/vasC-R & CTggTgATgTACgTgCTTACAAC \\
\hline vasD/vasE-F & AgATTgCgAATTAAgggACCAAA \\
\hline vasD/vasE-R & TgTCCgCCgATTACgACCA \\
\hline vasF/vasG-F & AAgCCTTCAATCATggCACTT \\
\hline vasF/vasG-R & TTATCAggCgCTgTTggAAT \\
\hline vasH/vasl-F & CCTgCgTCACTAACTCATTggA \\
\hline vasH/vasl-R & TTCAACCgTTgCCgATgggA \\
\hline vasl/vasJ-F & СТCTgCTCgACCTIgCgC \\
\hline vasl/vasJ-R & CgTgTigAACTggCgCTg \\
\hline vasK/vasL-F & TTGGTGTGAAACCACGGGAT \\
\hline vasK/vasL-R & CATCGGTGGAAGAGCTGAAG \\
\hline vasH-F1-up-Sall* & GCGTCGACGAATAAAACGGGAAGGCGAA \\
\hline vasH-F1-dn & TGCAATGCTGTTATGACAAGATCGCTATAT \\
\hline vasH-F2-up & CTTGTCATAACAGCATTGCATTTGAAAGCC \\
\hline vasH-F2-up-Sacl* & CGAGCTCAGAAACGGTGGTGCAGCTTG \\
\hline tssDI2c-F1-up-Sall* & GCGTCGACGCAATTCAATGGAGCGCCAA \\
\hline tssDI2c-F1-dn & ATITCATGA TGGCATCGTITTCCTTAG \\
\hline tssDI2c-F2-up & AACGATGCCA TCATGAAAATGACCCAGACT \\
\hline tssDI2c-F2-dn-Sacl* & CGAGCTCTTACAATGACACGATTCGCC \\
\hline tssD2a-F1-up-BamHI ${ }^{*}$ & CGGGATCCGCCAGCTITTACATCGC \\
\hline tssD2a-F1-dn & CGAGCGTAACTGCTCATTCCTTICTAACTG \\
\hline tssD2a-F2-up & GGAATGAGCAGTTACGCTCGAGCTGCGTTG \\
\hline tssD2a-F2-dn-Smal* & TCCCCCGGGAAACTGTAGTCTTGCAGC \\
\hline tssD2b-F1-up-Not| ${ }^{\star}$ & AAATATGCGGCCGCGCAAGCGTTCTCTGATCT \\
\hline tssD2b-F1-dn & CGAGCGTAACTGCTCATTCCTTTCTAACTG \\
\hline tssD2b-F2-up & GGAATGAGCAGTTACGCTCGAGCTGCGTTG \\
\hline tssD2b-F2-dn-Smal* & TCCCCCGGGTCTGGCTCACCTCCGATT \\
\hline
\end{tabular}

(Continued) 
TABLE 2 | Continued

\begin{tabular}{|c|c|}
\hline Primer & Oligonucleotide sequence $\left(5^{\prime}-3^{\prime}\right)$ \\
\hline VF-hcp-up-Ncol* & TGTCCATGGGCCCAACTCCATGTTATATCTCTATCG \\
\hline VF-hcp-dn-Xhol* & AGACTCGAGCGCTTCGATTGGTTACGCCA \\
\hline vasH-For-HindIII* & ССCAAGCTITCATAACGCCTTGATCTC \\
\hline vasH-Rev-Ndel ${ }^{*}$ & GGAATTCCATATGAGTAACTGGCTCGCT \\
\hline VF-recA-qPCR-up & ACCGAGTCAACGACGATAAC \\
\hline VF-recA-qPCR-dn & TGATGAACTGCTGGTGTCTC \\
\hline hcp-qPCR-F-com & TCGGCGATTCATTCGTT \\
\hline hcp-qPCR-R-com & CAGTTCAACCGTCGTCATCT \\
\hline VF-vasF-qPCR-F & СTGTGGCTCTTCCTCTTC \\
\hline VF-vasF-qPCR-R & TTATCAGTGCTTGGTGTTG \\
\hline VF-vasK-qPCR-F & ACATCCAACGCCAATACG \\
\hline VF-vasK-qPCR-R & CAATCGCAGTGAAGACAAC \\
\hline VF-vasH-qPCR-F & GGTAATCGGATACTGGAAC \\
\hline VF-vasH-qPCR-R & CATGTCAACTTGCTGGAT \\
\hline VF-tssD1-qPCR-F & TGTCGGTCACTCGTAACTC \\
\hline VF-tssD1-qPCR-R & TCAGAACCAGCACCATCAC \\
\hline vflB629-qPCR-F & GGTGGAAGTGTCTGGATGG \\
\hline vflB629-qPCR-R & TGGCTCAGGTTGGTATGC \\
\hline vflB643-qPCR-F & TGAACAACCGAGTGGCGAATATC \\
\hline vflB643-qPCR-R & CAGTTGGCGGAACGAGGATTG \\
\hline vflB631-qPCR-F & CGAGCCGAATATCCGTCTG \\
\hline vflB631-qPCR-R & TTCCTGCCGTTCCAACAC \\
\hline vflB647-qPCR-F & TTGCGGAAGTGATCTCTG \\
\hline vflB647-qPCR-R & TTACGGCTGTCGGTTAAG \\
\hline
\end{tabular}

*The underlined text indicates the restriction enzyme sites.

or pSRKTc ( $\Delta$ vasH/pSRKTc) were grown in the above LB with tetracycline to an $\mathrm{OD}_{600}$ of 0.5 . Each culture was then divided in half. One half was induced by the addition of IPTG (final concentration of $0.5 \mathrm{mM}$ ), and the other half (uninduced) was used as a control. The cultures were continually incubated until they reached an $\mathrm{OD}_{600}$ of 1.5 at $30^{\circ} \mathrm{C}$ with shaking.

Cells were pelleted at high speed in a tabletop microcentrifuge for $5 \mathrm{~min}$. Equal volumes of supernatants were filtered through $0.22 \mu \mathrm{m}$ low protein-binding polyvinylidine fluoride syringe filters (Millipore), and then a $1 / 100$ volume of $2 \%$ sodium deoxycholate was added, and the samples were incubated for 30 min on ice. Proteins were precipitated with $10 \%$ trichloroacetic acid (TCA) for $30 \mathrm{~min}$ on ice, pelleted by centrifugation at 12,000 rpm for $20 \mathrm{~min}$ at $4^{\circ} \mathrm{C}$, and washed twice with ice-cold acetone to remove residual TCA. Protein precipitates were resuspended in $40 \mu \mathrm{l}$ SDS-PAGE loading buffer $(10 \mathrm{mM}$ Tris- $\mathrm{HCl}, \mathrm{pH} 8.0$; 50 mM DTT; $1 \%$ SDS; $10 \%$ glycerol; $0.08 \%$ bromophenol blue) and boiled for $10 \mathrm{~min}$. Cell pellets were resuspended in PBS and the cell density was adjusted to $3.5 \mathrm{McF}$ arland standards. Then pellets from $1 \mathrm{ml}$ aliquots of above samples were suspended with $150 \mu \mathrm{l}$ of SDS-PAGE lysis buffer and boiled for $10 \mathrm{~min}$. Protein samples were separated by SDS-PAGE (12\%), transferred onto PVDF membranes, and analyzed by Western blotting using a rabbit anti-Hcp polyclonal antibody and an anti-E. coli CRP antibody (BioLegend, USA). The secondary antibodies used were horseradish peroxidase (HRP)-conjugated goat anti-rabbit and goat anti-mouse antibodies. Proteins were visualized using an Enhanced Chemiluminescence system (TaKaRa, Dalian, China). Each immunoblot experiment was repeated at least two times.

\section{RNA Extraction and qRT-PCR}

Total RNA extraction and quantitative reverse transcription (qRT)-PCR were performed as described previously (Wu et al., 2015). In brief, strain 85003 was grown to OD600 1.5 under the indicated conditions with agitation. Three independent samples were tested in triplicate. A control mixture using total RNA as a template was added in each reaction to exclude the effects of chromosomal DNA contamination. Relative expression values (R) were calculated using the equation $R=$

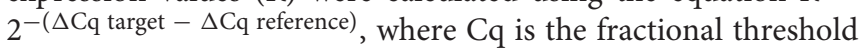
cycle. We used recA mRNA as a reference. The following primer combinations were used: VF-recA-qPCR-up and VFrecA-qPCR-dn for recA; hcp-qPCR-F-com and hcp-qPCR-R-com for $t s s D 2(h c p)$; VF-vasF-qPCR-F and VF-vasF-qPCR-R for $t s s L 2$ (vasF); VF-vasH-qPCR-F and VF-vasH-qPCR-R for vasH; VFvasK-qPCR-F and VF-vasK-qPCR-F for tssM2 (vasK); VF-tssD1qPCR-F and VF-tssD1-qPCR-R for tssD1; vflB629-qPCR-F and vflB629-qPCR-R for tss M1; vflB643-qPCR-F and vflB643-qPCR$\mathrm{R}$ for $t s s A 1$; vflB631-qPCR-F and vflB631-qPCR-R for $t s s K 1$ and vflB647-qPCR-F and vflB647-qPCR-R for rhs. Detailed primer sequence information is listed in Table 2.

\section{Bacterial Killing Assay}

V. fluvialis predator strains 85003 and $\Delta v a s H$, and E. coli prey MG1655, were grown overnight on LB agar containing $170 \mathrm{mM} \mathrm{NaCl}$ at $30^{\circ} \mathrm{C}$. Bacterial lawns were resuspended and normalized to $1.5 \mathrm{McF}$ arland standards in PBS and mixed at a 9:1 ratio (predator: prey) in triplicates. A total of $10 \mu \mathrm{l}$ of the mixtures were spotted on LB plates with $340 \mathrm{mM} \mathrm{NaCl}$ and incubated at $30^{\circ} \mathrm{C}$ for $5 \mathrm{~h}$. The colony forming units (CFU) per milliliter of the attacker and prey in the mixtures $(0 \mathrm{~h})$ were determined by plating 10 -fold serial dilutions on streptomycinand rifampicin-containing agar plates, respectively. Bacterial spots were harvested from LB plates after $5 \mathrm{~h}$, and the CFU per milliliter of the surviving attacker and prey $(5 \mathrm{~h})$ were determined on selective media plates as described above.

In the vasH complementation assay, IPTG was added to LB plates at a final concentration of $1 \mathrm{mM}$ to induce vasH expression. $V$. fluvialis strain $\Delta$ vas $\mathrm{H} / \mathrm{pSR}$ vas $H$ was grown overnight at $30^{\circ} \mathrm{C}$ on tetracycline-containing LB agar with $170 \mathrm{mM} \mathrm{NaCl}$ in the presence or absence of IPTG. The mixtures with prey were prepared as described above and spotted on LB plates containing $340 \mathrm{mM} \mathrm{NaCl}$ with and without IPTG. Strain $\Delta$ vasH/pSRKTc was used as a control.

\section{Nucleotide Sequences and Accession Numbers}

The sequences of VflT6SS1, VflT6SS2, and the three $h c p$ vgrG homologs from $V$. fluvialis 85003 were deposited in the NCBI database under accession numbers KY319183, KY319184, KY319185, KY319186, and KY319187, respectively. The genomic sequences of $V$. furnissii NCTC11218 (accession numbers NC_016602.1 and NC_016628.1), V. fluvialis 33809 
(accession numbers CP014034 and CP014035), V. cholerae N16961 (accession numbers NC_002505.1 and NC_002506.1) and $V$. splendidus LGP32 (accession numbers NC_011753.2 and NC_011744.2) were downloaded from the NCBI database.

\section{DNA and Protein Sequence Analysis}

The comparative analysis of T6SS sequences from $V$. fluvialis strains 85003 and 33809, V. cholerae N16961, V. furnissii NCTC11218 and V. splendidus LGP32 were performed using the BLAST software with an e-value of $1 \mathrm{e}-2$, and the alignments of $>1$ kilobase $(\mathrm{kb})$ were kept. VasH protein sequence alignments were performed with the GENEDOC program.

\section{RESULTS}

\section{Genetic Contents and Organization of T6SS in $V$. fluvialis}

Our previous draft genome analysis of $V$. fluvialis 85003 revealed the presence of putative homologs of T6SSs, which clustered and grouped into two genetic modules which are highly homologous to two separate regions on chromosome 2 of $V$. furnissii (Lu et al., 2014). In this study, we defined the detailed gene contents and organization of the two T6SSs, the flanking regions of the two T6SS clusters, and further identified two other "orphan" $h c p-v g r G$ homologs by PCR with primers designed based on the sequence of $V$. furnissii NCTC11218.

The cluster homologous to the $V$. furnissii vfu_B00799 vfu_B00780 was named VflT6SS1, and also shared partial synteny with the genetic region of VS_1337 VS_1318 of $V$. splendidus. In $V$. furnissii, three genes, vfu_B01185, vfu_B01189, and vfu_B01191, were predicted to encode T6SSrelated subunits $\mathrm{ClpB}$, IcmF-like protein and $\mathrm{VgrG}$, respectively. In V. splendidus, only VS_1326 was annotated to encode ClpB. The VflT6SS1 is a locus of around $24 \mathrm{~kb}$ consisting of 19 open reading frames (ORFs) with tight intergenic spaces and the same gene orientation. Homologs of V. splendidus VS_1324 and VS_1325 were not found in either $V$. furnissii or $V$. fluvialis. We designated 13 out of the 19 ORFs as VflT6SS1 tssA1-M1 (Type Six Secretion A1-M1) following the proposed nomenclature for T6SS components (Shalom et al., 2007; Figure 1). These showed $82-100 \%$ and $27-79 \%$ amino acid identity with the corresponding homologs of $V$. furnissii and $V$. splendidus, respectively. The VflT6SS1 cluster contains the T6SS hallmark gene, $t s s D(h c p)$, tssH (clpV/vasG) encoding an ortholog of the ClpB ATPase, which is considered important for T6SS function, and two additional genes, tssL (icmH/dotU/impK) and tss $M$ (icmF/impL), encoding homologs of T4SS stabilizing proteins (Boyer et al., 2009). Of note, the hcp of VflT6SS1 had only $40 \%$ nucleotide identity to the homologs in V. cholerae (VC1415 and VCA0017). To gain insight into the genetic background of VflT6SS1, its flanking regions were examined. The flanking sequence of 85003 VflT6SS1 was identical to that of $V$. furnissii NCTC 11218. The left junction of the sequences encodes a putative ABC-type transport periplasmic component and a MFS transporter, and the right junction possesses the sensor histidine kinase, BaeS. However, no homologous gene cluster of
VflT6SS1 was found in V. fluvialis strain ATCC 33809, a newly whole-genome sequenced $V$. fluvialis isolate from Bangladesh.

The cluster homologous to the $V$. furnissii vfu_B01176 vfu_B01191 genomic region was named VflT6SS2. It spans $21.22 \mathrm{~kb}$ and contains 16 ORFs which display the same organization and gene orientation as the "core" gene cluster of $V$. cholerae T6SS (VCA0107 VCA0124). The major characteristics of the VflT6SS2 gene cluster of $V$. fluvialis strain 85003 and its most closely related orthologs in other species are summarized in Table 3.

VflT6SS2 is located between the usp and $r s b D$ genes in 85003, the same as in $V$. cholerae and $V$. furnissii, indicating its conserved location. Apart from the core gene cluster, $V$. cholerae T6SS has two copies of $h c p-v g r G$ alleles encoded in two small auxiliary clusters (VCA0017 VCA0021 and VC1415 VC1421). In the 85003 draft genome, we only identified one copy of the $h c p$-vgrG homolog and the counterparts of vfu_B001191 and VCA0124 (vgrG3) from V. furnissii and V. cholerae, respectively, were missing in the originally aligned VflT6SS2 genetic locus. Sequence alignments showed that the two copies of hcp-vgrG modules exist in similar genetic locations in both $V$. furnissii and $V$. cholerae. Therefore, we reasoned that undiscovered $h c p$ $\operatorname{vgrG}$ copies likely existed in $V$. fluvialis 85003. V. fluvialis and $V$. furnissii are the genetically closest species among Vibrionaceae, with a mean BLASTp identity of $86.8 \%$ for the homologous genes (Lu et al., 2014). V. furnissii was originally regarded as an aerogenic biogroup of $V$. fluvialis and was later confirmed to be a separate species from $V$. fluvialis (Brenner et al., 1983). Using primer pairs vfu-vasL-up2153177/vfurbsD-dn2158250, vfuA01956-up/vfuA01959-dn, and vfuB01009up/vfuB01011-dn, which were designed on the basis of the flanking sequences of $V$. furnissii vfu_B01191 vfu_B01192 and the two hcp-vgrG modules (vfu_A01957 vfu_A01958 and vfu_B01010 vfu_B01011, respectively), we amplified the corresponding regions of 85003 with long fragment PCR.

Primer pair vfu-vasL-up2153177/vfu-rbsD-dn2158250 yielded a 5093 bp fragment for $V$. fluvialis 85003. A sequence analysis of the fragment revealed a 3039 bp ORF which had 82 and 60\% nucleotide identity to vfu_B001191 and VCA0123 ( $\operatorname{grG} 3$ ), respectively. Additionally, a 384 bp ORF was predicted immediately downstream and has 4 bp overlap with the 3,039 bp ORF. This organizational feature was similar to VCA0123 and VCA0124, which were experimentally demonstrated to encode a $\operatorname{vgrG} 3$ effector and its immunity effector, TsiV3, in $V$. cholerae (Dong et al., 2013). Thus, we named the two ORF tssI2 and tsiI2, respectively. A sequence analysis and alignment of the 3,145 and 3,878 bp PCR products of primer pair vfuA01956up/vfuA01959-up and vfuB01009-up/vfuB01011-dn indicated that each encodes a copy of the hcp-vgrG homolog, which was different from the originally recognized $h c p-v g r G$ allele. In order to distinguish them, we designated the newly-identified alleles as tssD2_a-tssI2_ $a$ and $t s s D 2 \_b$-tssI2_b (corresponding to $V$. cholerae hcp1-vgrG1 and hcp2-vgrG2, respectively), and the original one was named tssD2_c-tssI2_c. The three copies of TssD (Hcp) show high identity (99.42\%) in their nucleotide sequences and have $95.35 \%$ amino acid identity to the well-characterized Hcp protein of $V$. cholerae. TssI2_a and TssI2_b display higher 

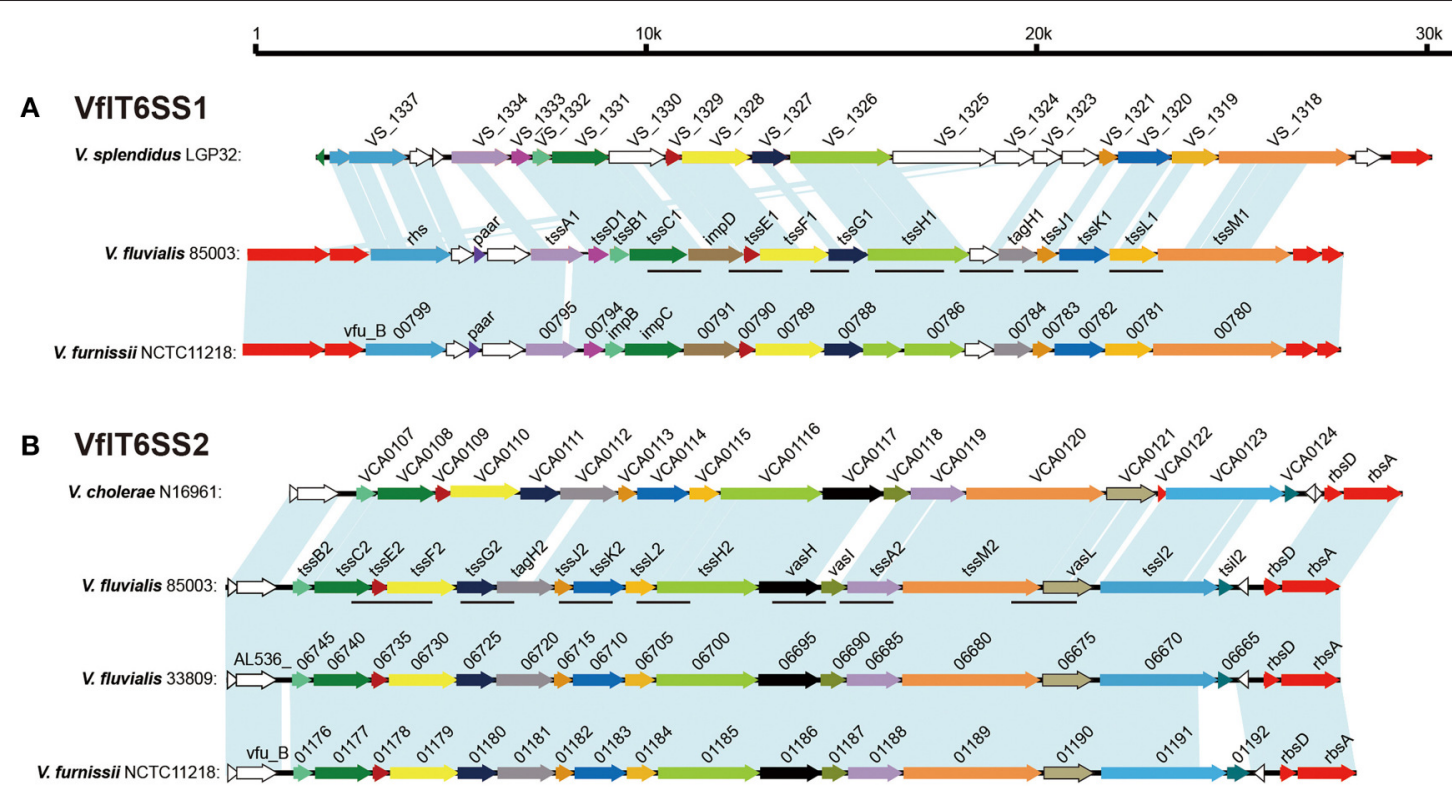

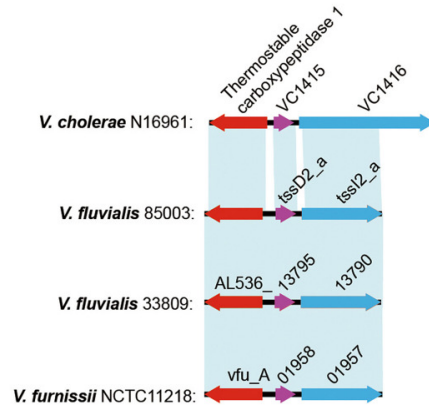

tssD2_a-tss/2_a

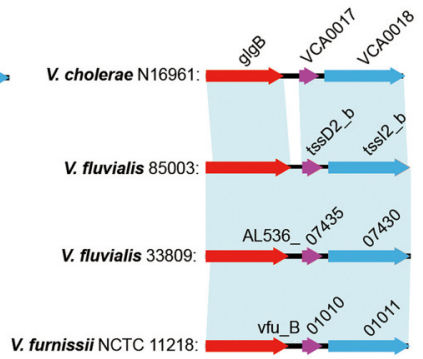

tssD2_b-tssI2_b

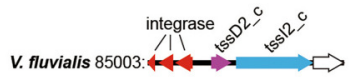

tssD2_c-tss/2_c

FIGURE 1 | Gene organization of the T6SS clusters in $\boldsymbol{V}$. fluvialis in comparison with $\boldsymbol{V}$. furnissii, $\boldsymbol{V}$. cholerae, and $\boldsymbol{V}$. splendidus. (A) Genes encoding VfIT6SS1 in V. fluvialis 85003 were named according to the Tss nomenclature (tssA1, tssB1, tssC1, tssD1, tssE1, tssF1, tssG1, tss $H 1$, tss 1 , tssK 1, tss $L 1$, tss 1 1, tagH1) or by their usual vernacular names (paar, impD, and rhs). Homologous genes are colored similarly. Open reading frames with unknown functions are shown in white. The lines below the colored genes indicate the loci amplified by PCR in the different isolates. (B) Genes encoding VflT6SS2 in V. fluvialis 85003 were names according to the Tss nomenclature (tssA2, tssB2, tssC2, tssE2, tssF2, tssG2, tssH2, tss/2, tssJ2, tssK2, tss L2, tssM2, tagH2) or vernacular names (vasH, vasl, tsil2, and vasL). The 17 genes show high conservation with the well-characterized T6SS of $V$. cholerae N16961. The counterparts of $V$. fluvialis 33809 and $V$. furnissii NCTC11218 exhibit the identical gene organization in their respective chromosomes. The lines below the colored genes indicate the loci amplified by PCR in the different isolates. Three "orphan" hcp-vgrG alleles were designated tssD2_a-tss/2_a, tssD2_b-tss/2_b, and tssD2_c-tss/2_c, respectively. The homolog of tssD2_c-tss/2_c is absent in V. fluvialis 33809, V. furnissii NCTC11218, and V. cholerae N16961.

homology with up to $88 \%$ amino acid identity. TssI2_c shows 60 and $61 \%$ identity to TssI2_a and TssI2_b respectively. The corresponding sequences of these three $h c p$-vgrG alleles were deposited in GenBank under accession numbers KY319185, KY319186, and KY319187, respectively.

\section{Prevalence of $V$. fluvialis T6SS}

In many species, T6SS gene clusters are located on pathogenicity islands or compositionally distinct regions of the genome indicating its horizontal acquisition and transfer. To better understand the gene content, sequence similarity, synteny, and the distribution of T6SS clusters in $V$. fluvialis, we carried out PCR screening of each T6SS locus in 34 different $V$. fluvialis isolates from both human and aquatic products from different provinces in China. The genetic loci of tssC1 (impC)-impD, tssE1 (impF)-tssF1 (impG), tssF1 (impG)-tssG1 (impH), tssH1, tssH1 (clpB)-tagH1 (impI), tagH1-tssK1 (impJ), and tssL1 (impK)tssM1 (impL) within VflT6SS1 were all detected in seven out of 34 tested isolates (21\% detection rate). The genetic loci of $t s s C 2$ (vipB)-tssF2 (vasA), tssG2 (vasB)-tagH2 (vasC), tss 2 (vasD)tssK2 (vasE), tssL2 (vasF)-tssH2 (vasG), vasH-vasI, vasI-tss $A 2$ (vasJ), and tssM2 (vasK)-vasL for VflT6SS2 were detected in 100, 97, 97, 100, 91, 94, and 94\% of the isolates, respectively. These results revealed that in general, VflT6SS2 is more prevalent than VflT6SS1 in $V$. fluvialis. The different detection rates of VflT6SS2 component gene loci may indicate that there is variation in the gene composition of in the T6SS2 cluster or sequence variation from the corresponding primer annealing regions in different 
TABLE 3 | Characteristics of VfIT6SS2 components and the most closely related orthologs in other species.

\begin{tabular}{|c|c|c|c|c|c|c|}
\hline \multicolumn{3}{|c|}{ V. fluvialis 85003} & \multirow[t]{2}{*}{ Homologs } & \multirow{2}{*}{$\begin{array}{l}\text { V. cholerae N16961 } \\
\text { Gene ID (identity\%) }\end{array}$} & \multirow{2}{*}{$\begin{array}{l}\text { V. furnissii NCTC11218 } \\
\text { Gene ID (identity\%) }\end{array}$} & \multirow{2}{*}{$\begin{array}{l}\text { V. fluvialis } 33809 \\
\text { Gene ID (identity\%) }\end{array}$} \\
\hline Gene & Size (bp) & COG & & & & \\
\hline tssB2 & 510 & 3,516 & impB, vipA & VCA0107 (87.88) & vfu_B01176 (96.47) & AL536_06745 (98.82) \\
\hline tssC2 & 1,476 & 3,517 & impC, vipB & VCA0108 (92.68) & vfu_B01177 (98.58) & AL536_06740 (98.37) \\
\hline tssE2 & 438 & 3,518 & impF, vasS, hsiF & VCA0109 (89.73) & vfu_B01178 (100.00) & AL536_06735 (99.31) \\
\hline tssF2 & 1,770 & 3,519 & impG, vasA & VCA0110 (84.92) & vfu_B01179 (98.47) & AL536_06730 (99.83) \\
\hline tssG2 & 1,017 & 3,520 & $i m p H$, vasB & VCA0111 (79.35) & vfu_B01180 (96.95) & AL536_06725 (99.70) \\
\hline $\operatorname{tag} \mathrm{H} 2$ & 1,479 & 3,456 & impl, vasC, fha & VCA0112 (64.86) & vfu_B01181 (91.73) & AL536_06720 (97.76) \\
\hline tss 2 & 477 & 3,521 & lip, vas D & VCA0113 (84.91) & vfu_B01182 (98.74) & AL536_06715 (98.11) \\
\hline tssK2 & 1,335 & 3,522 & impJ, vasE & VCA0114 (90.34) & vfu_B01183 (98.88) & AL536_06710 (97.30) \\
\hline tssL2 & 774 & 3,455 & impK, vasF, icmH/dotU, & VCA0115 (86.05) & vfu_B01184 (99.61) & AL536_06705 (100.00) \\
\hline tssH2 & 2,613 & 0,542 & clpV, vasG & VCA0116 (86.09) & vfu_B01185 (97.47) & AL536_06700 (96.79) \\
\hline vasH & 1,596 & 1,221 & sfa & VCA0117 (73.82) & vfu_B01186 (96.11) & AL536_06695 (98.5) \\
\hline vasl & 660 & - & vasl & VCA0118 (47.81) & vfu_B01187 (86.76) & AL536_06690 (95.91) \\
\hline tssA2 & 1,401 & 3,515 & impA, vasJ & VCA0119 (70.42) & vfu_B01188 (95.29) & AL536_06685 (98.72) \\
\hline tssM2 & 3,546 & 3,523 & impL, icmF, vasK & VCA0120 (85.19) & vfu_B01189 (98.48) & AL536_06680 (100) \\
\hline vast & 1,278 & 3,515 & vasL & VCA0121 (55.45) & vfu_B01190 (93.43) & AL536_06675 (98.35) \\
\hline tss/2 & 3,039 & 3,501 & vgrG & VCA0123 (54.03) & vfu_B01191 (73.74) & AL536_06670 (97.00) \\
\hline tsil2 & 384 & - & tsiv3 & VCA0124 & vfu_B01192 & AL536_06665 (94.51) \\
\hline tssD2_a & 519 & 3,157 & $h c p$ & VC1415 (95.38) & vfu_A01958 (99.42) & AL536_13795 (100.00) \\
\hline tssl2_a & 2,067 & 3,501 & $\operatorname{vgrG}$ & VC1416 (44.58) & vfu_A01957 (96.37) & AL536_13790 (96.22) \\
\hline tssD2_b & 519 & 3,157 & $h c p$ & VCA0017 (95.38) & vfu_B01010 (99.42) & AL536_07435 (100.00) \\
\hline tssl2_b & 2,097 & 3,501 & $\operatorname{vgrG}$ & VCA0018 (74.24) & vfu_B01011 (97.28) & AL536_07430 (97.14) \\
\hline tssD2_c & 519 & 3,157 & $h c p$ & - & - & - \\
\hline tssl2_c & 1,980 & 3,501 & $\operatorname{vgrG}$ & - & - & - \\
\hline
\end{tabular}

-, Unknown or does not exist.

isolates. These variations need to be investigated in greater detail in future studies. Since we found that VflT6SS2 is highly prevalent in $V$. fluvialis strains, we first focused on the functional characterization of VflT6SS2 in V. fluvialis 85003.

\section{Determination of the Functional Expression of $\boldsymbol{V}$. fluvialis VfIT6SS2}

The hallmark of a functional T6SS is the presence of Hcp and VgrG in the culture supernatant. Therefore, we examined the Hcp secretion in $V$. fluvialis cultures. To this end, we first cloned and expressed $h c p$ in pET30a with induction by IPTG. The purified Hcp protein was used to prepare anti-Hcp polyclonal antiserum in rabbits, because there is no commercially-available antibody against this protein.

To determine whether VflT6SS2 was functionally expressed, clinical isolates 85003, VF42, and CICC21612, and environmental isolate VF54, all of which contain the complete VflT6SS2 based on a PCR screen, were routinely cultured in $\mathrm{LB}$ with $170 \mathrm{mM} \mathrm{NaCl}$ at $30^{\circ} \mathrm{C}$. The growth curves of VF42, VF54, and CICC21612 displayed consistent growth trends before entering a stationary growth phase, while 85003 grew slowly relative to these other strains (Figure S1). However, all four strains were in the logarithmic growth phase at an OD600 of 1.5. The cell pellets and culture supernatants of each strain at an $\mathrm{OD}_{600}$ of 1.5 were examined by immunoblot analyses using polyclonal anti-Hcp antiserum. As shown in Figure 2, a large amount of Hcp was detected in both the cell pellets and culture supernatants of 85003, CICC21612, and VF54, indicating the functional expression of T6SS2 in these $V$. fluvialis strains. Of note, the absence of the cytosolic Crp in the supernatants demonstrated that the bacteria were intact, and thus, Hcp was being actively secreted and not released by cell lysis. This control was routinely used in the subsequent Hcp secretion analysis. For strain VF42, Hcp was only detected in the cell pellets, indicating that, though Hcp was synthesized in these bacteria, it was not efficiently secreted into the supernatant. Taken together, our results demonstrated that functional T6SS exists in at least some $V$. fluvialis isolates.

It's should be noted that the apparent molecular weight of Hcp in the Western blot analysis (around $25 \mathrm{kDa}$ ) is quite different from the predicted size according to its ORF sequence $(19 \mathrm{kDa})$. This inconsistency has also been observed in $V$. cholerae (Ishikawa et al., 2009) and V. alginolyticus (Sheng et al., 2012), and possible modification of two cysteine residues of Hcp at positions 5 and 22 was suggested to be responsible for the difference (Williams et al., 1996).

\section{Growth Phase-Dependent Secretion of Hcp from V. fluvialis VfIT6SS2}

Following the confirmation of the functional expression of VflT6SS2 in $V$. fluvialis, we tested the effects of the bacterial growth phase on the expression and secretion of Hcp. Strain 


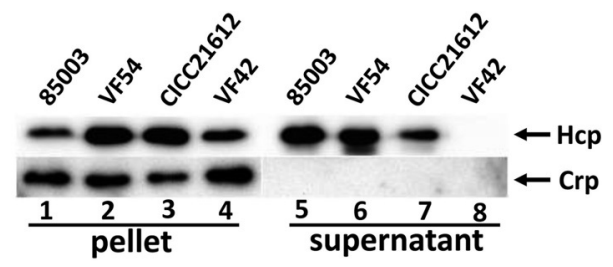

FIGURE 2 | Immunoblot analyses of VfIT6SS2 Hcp in cell pellets and supernatants from $\boldsymbol{V}$. fluvialis isolates. The bacterial strains were grown in LB with $170 \mathrm{mM} \mathrm{NaCl}$ at $30^{\circ} \mathrm{C}$ to an $\mathrm{OD}_{600}$ of 1.5. SDS-PAGE and immunoblot analyses were performed using anti-Hcp and anti-Crp antibodies. Lanes 1-4, cell pellets; lanes 5-8, culture supernatants of $V$. fluvialis strains 85003, VF54, VF42, and CICC21612, respectively. The arrows show the immunoblot band to Hcp and Crp. The Crp protein is absent in the culture supernatants, indicating that the detection of $\mathrm{Hcp}$ in the supernatants was not a consequence of cell lysis.

85003 was grown in $\mathrm{LB}$ medium containing $170 \mathrm{mM} \mathrm{NaCl}$ at $30^{\circ} \mathrm{C}$, and the protein levels of Hcp in the cell pellets and culture supernatants were measured at different growth points (i.e., $\mathrm{OD}_{600} 0.2,0.5,1.0,1.5,2.0,2.5,3.8$, and overnight; Figures 3A,B). Hcp expression was detected in the cell pellets at all growth points except overnight, and the expression level at an $\mathrm{OD}_{600}$ of 0.2 was obviously lower than that of the other growth points (Figure 3B, lane 1). However, the secretion of Hcp was only detected at $\mathrm{OD}_{600}$ values of $1.0,1.5$, and 2.0 , and the highest secretion level was observed at an $\mathrm{OD}_{600}$ of 1.5 (Figure 3B, lanes 11-13). Our results indicated that, although the Hcp was continuously synthesized at various $\mathrm{OD}_{600}$ values (except overnight culture), the active T6SS was only detected during a narrow window of the growth phase. It appears that when the cell density reaches an $\mathrm{OD}_{600}$ of 2.5 , the secretion of Hcp stops. At the later stationary phase after overnight incubation, when no Hcp was detected, the expression of $h c p$ may have been turned off or Hcp may have been proteolytically degraded.

\section{Temperature-Dependent Expression and Secretion of Hcp by VfIT6SS2}

Like many species of Vibrios, V. fluvialis can survive under different environmental conditions, from the aquatic milieu, such as seas, estuaries, and brackish waters, to the human intestine. We wondered whether the temperature affects the function of VflT6SS2 in V. fluvialis. Therefore, we cultured the 85003 strain at different temperatures $\left(25^{\circ}, 30^{\circ}\right.$, or $\left.37^{\circ} \mathrm{C}\right)$ and compared the expression and secretion of Hcp. Although 85003 grows faster at $37^{\circ} \mathrm{C}$ than at $25^{\circ}$ and $30^{\circ} \mathrm{C}$ (Figure S2), the $\mathrm{OD}_{600}$ of 1.5 lies at the same log growth phase. Hcp was expressed and secreted at both $25^{\circ} \mathrm{C}$ and $30^{\circ} \mathrm{C}$, but not at $37^{\circ} \mathrm{C}$ (Figure $4 \mathrm{~A}$ ). The secretion of $\mathrm{Hcp}$ at $30^{\circ} \mathrm{C}$ was higher than that at $25^{\circ} \mathrm{C}$ (Figure $4 \mathrm{~A}$, compare lane 4 with lane 5). Our results revealed that VflT6SS2 is active under cool and warm temperature, similar to T6SS2 in $V$. parahaemolyticus, which is most active at cold $\left(23^{\circ} \mathrm{C}\right)$ and warm $\left(30^{\circ} \mathrm{C}\right)$ temperatures under low salt conditions (Salomon et al., 2013), suggesting that VflT6SS2 may play a role in the environmental fitness of $V$. fluvialis.

To explore the molecular mechanism(s) responsible for the undetectable protein level of $\mathrm{Hcp}$ at $37^{\circ} \mathrm{C}$, we measured the

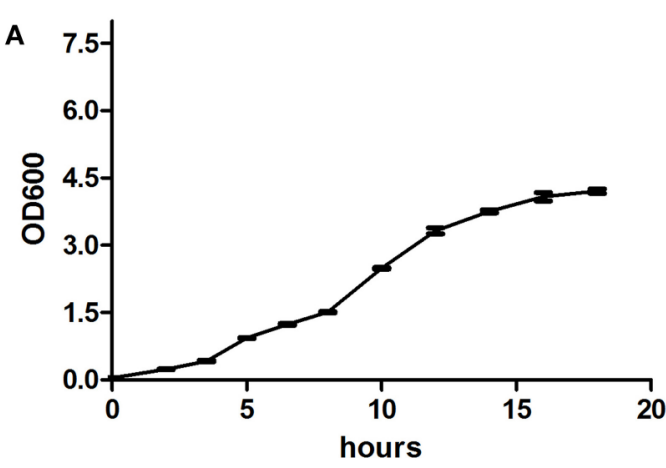

B

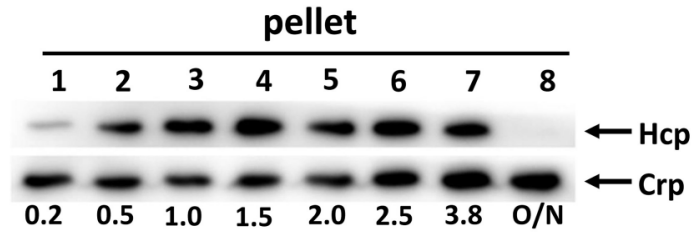

$\mathrm{OD}_{600}$

$\begin{array}{llllllll}0.2 & 0.5 & 1.0 & 1.5 & 2.0 & 2.5 & 3.8 & 0 / N\end{array}$

supernatant

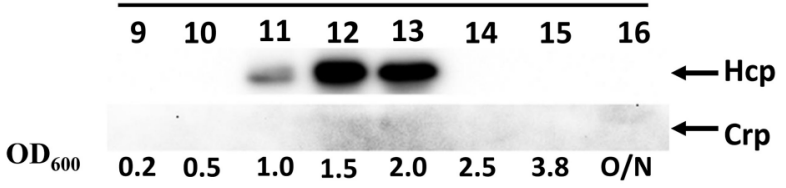

FIGURE 3 | VfIT6SS2 Hcp levels in V. fluvialis 85003 at different parts of the growth phase. (A) Growth curve of $V$. fluvialis strain 85003 incubated in LB media containing $170 \mathrm{mM} \mathrm{NaCl}$ at $30^{\circ} \mathrm{C}$. Error bars indicate the standard deviation of three independent cultures. (B) Immunoblot analysis of $\mathrm{Hcp}$ expression. The $V$. fluvialis strain 85003 was grown in LB containing $170 \mathrm{mM}$ $\mathrm{NaCl}$ at $30^{\circ} \mathrm{C}$ and the cell pellet and culture supernatant samples were taken at different parts of the growth phase $\left(\mathrm{OD}_{600 \mathrm{~nm}} 0.2,0.5,1.0,1.5,2.0,2.5\right.$, 3.8, and overnight). SDS-PAGE and immunoblot analyses were performed using anti-Hcp and anti-Crp antibodies. Lanes 1-8, cell pellets; lanes 9-16, culture supernatants. The arrows indicate the reaction bands of the Hcp and Crp proteins.

transcription levels of $t s s \mathrm{D} 2(h c p)$, together with three selected VflT6SS2 core genes [tssL2 (vasF), vasH, and tssM2 (vasK)] by qRT-PCR using cultures grown at $30^{\circ}$ and $37^{\circ} \mathrm{C}$. As shown in Figure 4B, the tss $\mathrm{D} 2(h c p)$ mRNA level was dramatically decreased (more than 150 -fold) at $37^{\circ} \mathrm{C}$ compared to that at $30^{\circ} \mathrm{C}$. However, no considerable alterations were observed in the mRNA levels of $t s s L 2$ (vasF), vasH, and tss 22 (vasK) between $30^{\circ}$ and $37^{\circ} \mathrm{C}$, suggesting that the loss of function of VflT6SS2 at $37^{\circ} \mathrm{C}$ is mainly due to the extremely low transcription level of the $t s s D 2$ (hcp) gene at this temperature.

\section{Salinity and Osmolarity-Dependent Expression and Secretion of Hcp from VfIT6SS2}

As V. fluvialis is a halophilic species of Vibrio and normally resides in coastal and estuarine environments, we investigated if the salinity influences the expression and secretion of Hcp in V. fluvialis. We cultured strain 85003 at $30^{\circ} \mathrm{C}$ to an $\mathrm{OD}_{600}$ of 1.5 in $\mathrm{LB}$ containing increasing concentrations of $\mathrm{NaCl}$. We 

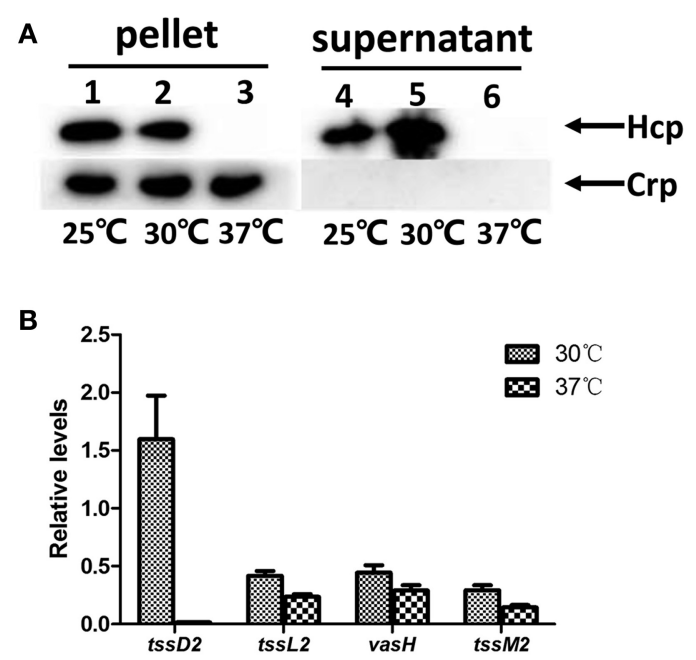

FIGURE 4 | Effects of temperature on the expression of VfIT6SS2 by $V$. fluvialis 85003. (A) Immunoblot analysis of $\mathrm{Hcp}$ expression. The $V$. fluvialis strain 85003 was grown in LB containing $170 \mathrm{mM} \mathrm{NaCl}$ to an $\mathrm{OD}_{600}$ of 1.5 at $25^{\circ}, 30^{\circ}$, or $37^{\circ} \mathrm{C}$. Cell pellets and corresponding supernatants were analyzed by SDS-PAGE and immunoblot assays using anti-Hcp and anti-Crp antisera. Lanes 1-3, cell pellets; lanes 4-6, culture supernatants. The arrows indicate the reaction bands of the Hcp and Crp proteins. (B) qRT-PCR analysis of the mRNA abundance of VflT6SS2 genes. The $V$. fluvialis strain 85003 was grown in LB containing $170 \mathrm{mM} \mathrm{NaCl}$ to an $\mathrm{OD}_{600}$ of 1.5 at $30^{\circ}$ or $37^{\circ} \mathrm{C}$. RNA was extracted, and the abundances of tssD2, tss $L 2$, vas $\mathrm{H}$, and tss $\mathrm{M} 2$ mRNA were determined by qRT-PCR as described in the Materials and Methods. Each value is the average for three independent cultures. The error bars indicate standard deviations from the mean.

observed that comparable amounts of Hcp were detected in the cell pellets regardless of the salt concentration (Figure 5A, left panel). Significant amounts of secreted Hcp were readily detected in supernatants from cultures containing 170, 340, 513 , or $855 \mathrm{mM} \mathrm{NaCl}$, but not in supernatants from cultures containing $85 \mathrm{mM} \mathrm{NaCl}$ (Figure 5A, right panel, compare lane 6-lanes 7-10), although the secretion levels at 513 and $855 \mathrm{mM}$ $\mathrm{NaCl}$ appeared to be slightly reduced compared to those at 170 and $340 \mathrm{mM} \mathrm{NaCl}$. These results indicated that Hcp secretion was stimulated by high $\mathrm{NaCl}$ concentrations. To obtain more detailed information, we investigated whether salinity can influence the expression of $t s s D 2$ and the VflT6SS2 core gene cluster. As shown in Figure 5B, tssD2 and the all three selected VflT6SS2 core genes, $t s s L 2, v a s H$, and $t s s M 2$, were apparently induced when strain 85003 was grown at $30^{\circ} \mathrm{C}$ in $\mathrm{LB}$ containing $340 \mathrm{mM} \mathrm{NaCl}$ compared to cultures grown with $85 \mathrm{mM} \mathrm{NaCl}$.

We then determined whether the salinity could also affect VflT6SS2 at $37^{\circ} \mathrm{C}$. We therefore cultured the 85003 strain at $37^{\circ} \mathrm{C}$ in $\mathrm{LB}$ containing different concentrations of $\mathrm{NaCl}$ and analyzed the expression and secretion of Hcp. We found that steadily increasing amounts of Hcp were detected in pellets from cultures supplemented with 340,513 , or $855 \mathrm{mM} \mathrm{NaCl}$ (Figure 5C, lanes 3-5), and the secreted form of Hcp was detected under 513 or $855 \mathrm{mM} \mathrm{NaCl}$ conditions (Figure 5C, lanes 910). On the basis of these results, we inferred that high $\mathrm{NaCl}$ concentrations could induce both the expression and secretion

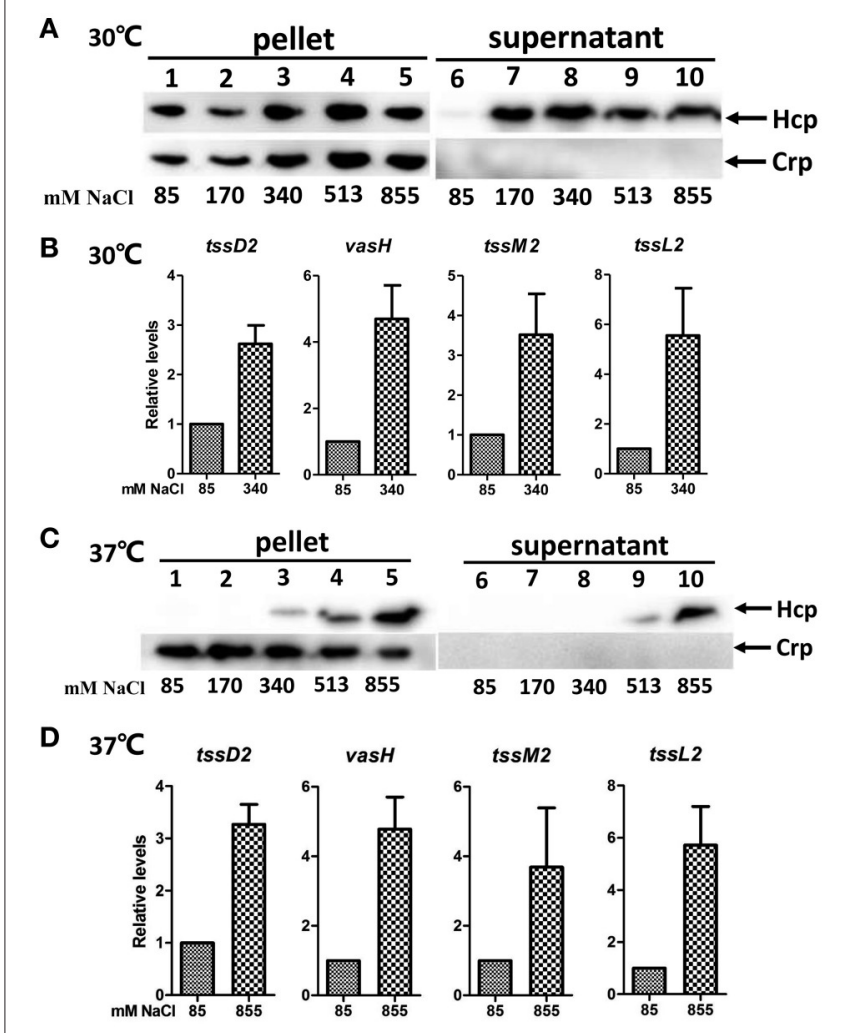

FIGURE 5 | Effects of salinity on the expression of VfIT6SS2 Hcp by $\boldsymbol{V}$. fluvialis 85003. (A,C) Immunoblot analysis of $\mathrm{Hcp}$ expression. The $\mathrm{V}$. fluvialis strain 85003 was grown at $30^{\circ}$ or $37^{\circ} \mathrm{C}$ in LB containing the indicted concentrations of $\mathrm{NaCl}$. Cell pellets and culture supernatants were analyzed by SDS-PAGE and immunoblot assays using anti-Hcp and anti-Crp antisera. Lanes 1-5, cell pellets; lanes 6-10, culture supernatants. The arrows indicate the reaction bands of the Hcp and Crp proteins. (B,D) qRT-PCR analysis of the mRNA abundance of VfIT6SS2 genes. The $V$. fluvialis strain 85003 was grown at $30^{\circ}$ or $37^{\circ} \mathrm{C}$ in LB containing the indicted concentrations of $\mathrm{NaCl}$. RNA was extracted, and the mRNA abundances of tss D2, tss $L 2$, vas $H$, and tssM2 were determined by qRT-PCR as described in the Materials and Methods. Data from three independent cultures were plotted to show the relative levels of transcripts, with the average level from low $(85 \mathrm{mM} \mathrm{NaCl})$ salinity samples in each case set to 1.0. The error bars indicate standard deviations from the mean.

of Hcp in a dose-dependent manner at $37^{\circ} \mathrm{C}$. Consistently, increased mRNA levels of $t s s D 2, t s s L 2, v a s H$, and $t s s M 2$ were confirmed when the 85003 strain was grown at $37^{\circ} \mathrm{C}$ in $\mathrm{LB}$ containing $855 \mathrm{mM} \mathrm{NaCl}$ (Figure 5D). These results indicated that, under certain circumstances, such as high salinity, VflT6SS2 would be activated in an infected host. The precise stimuli and signaling mechanism(s) involved in this response should be further investigated in future studies.

In $V$. cholerae A1552, it was reported that Hcp secretion was induced by osmolarity rather than salinity (Ishikawa et al., 2012). To test whether this is also the case in V. fluvialis, strain 85003 was grown at $30^{\circ} \mathrm{C}$ in $\mathrm{LB}$ supplemented with mannitol, trehalose or sucrose at a concentration of $510 \mathrm{mM}$, or $\mathrm{KCl}$ at a concentration of $255 \mathrm{mM}$, in addition to $85 \mathrm{mM} \mathrm{NaCl}$ to yield an equivalent osmolarity as $340 \mathrm{mM} \mathrm{NaCl}$. Based on the growth curve (Figure 6A), we collected samples from cultures 
with an $\mathrm{OD}_{600}$ of 1.5. Our results demonstrated that although the protein levels of Hcp in pellets were comparable under low and high osmolarity conditions (Figure 6B, left panel), the protein was more efficiently secreted under high osmolarity conditions (Figure 6B, compare lane 7 with lanes 8-12). Furthermore under the same osmolarity conditions, $\mathrm{NaCl}$, mannitol and trehalose seemed to more potently enhance the secretion of Hcp than sucrose and $\mathrm{KCl}$ (Figure 6B, compare lanes 8, 9, 11 with lanes 10 and 12). Together, these results indicate that high osmolarity induces Hcp secretion in V. fluvialis, and the osmolaritydependent secretion of Hcp may be a common characteristic among Vibrio species.

\section{V. fluvialis VfIT6SS2 Plays a Role in Interbacterial Virulence}

To test if VflT6SS2 activity contributes to interbacterial virulence or competition, we performed an E. coli killing assay. We first generated a $v a s H$ in-frame deletion mutant, $\Delta v a s H$, which was used as an avirulent control. VasH is considered to be a global transcriptional regulator of T6SS and is required to initiate the transcription of T6SS genes in V. cholerae (Kitaoka et al., 2011). The VasH of $V$. fluvialis encodes 531 amino acids and shares $73 \%$ identity with that of $V$. cholerae. Of note, the Walker A (GETGTGKE) motif, Walker B (GTLFLDEIG) motif, and the central GAFSGA-loop 1 region of the ATPase domain of VasH are exactly the same in $V$. cholerae and $V$. fluvialis (Figure S3). Consistent with the findings in $V$. cholerae, the deletion of vasH in $V$. fluvialis resulted in a lack of expression and secretion of Hcp

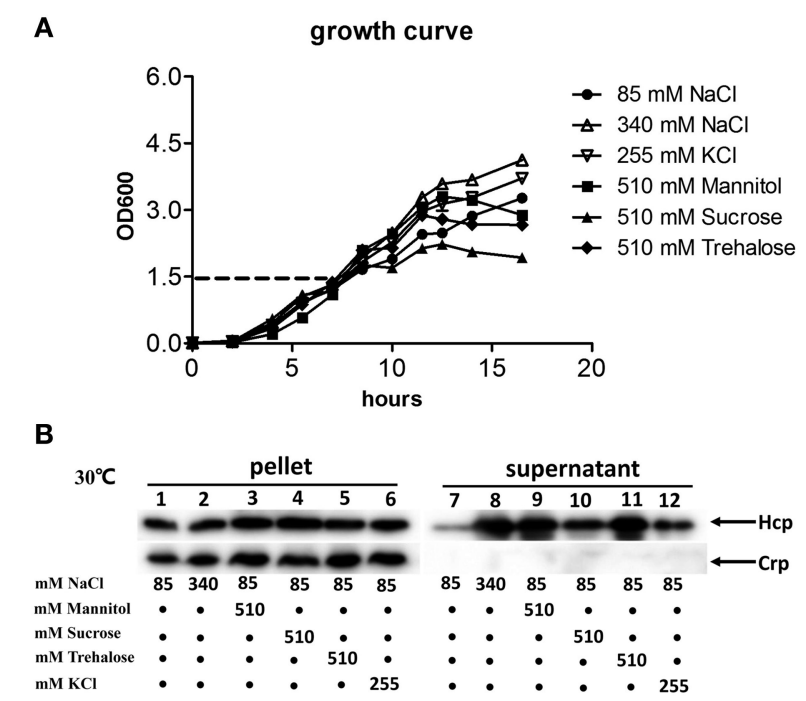

FIGURE 6 | Effects of osmolarity on the expression and secretion of VfIT6SS2 Hcp from V. fluvialis 85003. (A) Growth curves of $V$. fluvialis strain 85003 incubated at $30^{\circ} \mathrm{C}$ in normal LB media ( $85 \mathrm{mM} \mathrm{NaCl}$ ) or normal LB supplemented with different amounts of salt ( $\mathrm{NaCl}$ or $\mathrm{KCl}$ ) or sugar alcohol (mannitol, sucrose or trehalose) to yield the same osmolarity as $340 \mathrm{mM} \mathrm{NaCl}$. Error bars indicate the standard deviation of three independent cultures. (B) Immunoblot analysis of Hcp expression and secretion. The $V$. fluvialis strain 85003 was grown as described above. Lanes 1-6, cell pellets; lanes 7-12, culture supernatants.
(Figure 7A, compare lane 1 to lane 2, and lane 7 to lane 8), i.e., the naturally functional VflT6SS2 was completely disabled. However, the expression and secretion of Hcp were restored by introducing a complemented plasmid pSRvasH under IPTG induction, but the restoration did not occur if the pSRKTc control plasmid was used or no IPTG induction was employed (Figure 7A). The mixtures of the prey E. coli K-12 MG1655 and V. fluvialis predators were spotted on LB nutrient agar plates containing $340 \mathrm{mM} \mathrm{NaCl}$ and incubated at $30^{\circ} \mathrm{C}$. The number of surviving MG1655 was determined after $5 \mathrm{~h}$ of incubation. The results showed that the survival of MG1655 was significantly reduced in the presence of wild-type $V$. fluvialis, but not when vasH was deleted (Figure 7B). Re-expression of vasH from pSRvasH recovered the ability of $V$. fluvialis to compete against the E. coli, although not to the level of the wild type (Figure 7B). The above results collectively indicate that VflT6SS2 provides $V$. fluvialis with increased competitive fitness.

To determine whether VflT6SS1 is involved in the competitive bacterial killing demonstrated by $V$. fluvialis, we measured the protein level of TssD1, as well as the mRNA levels of three

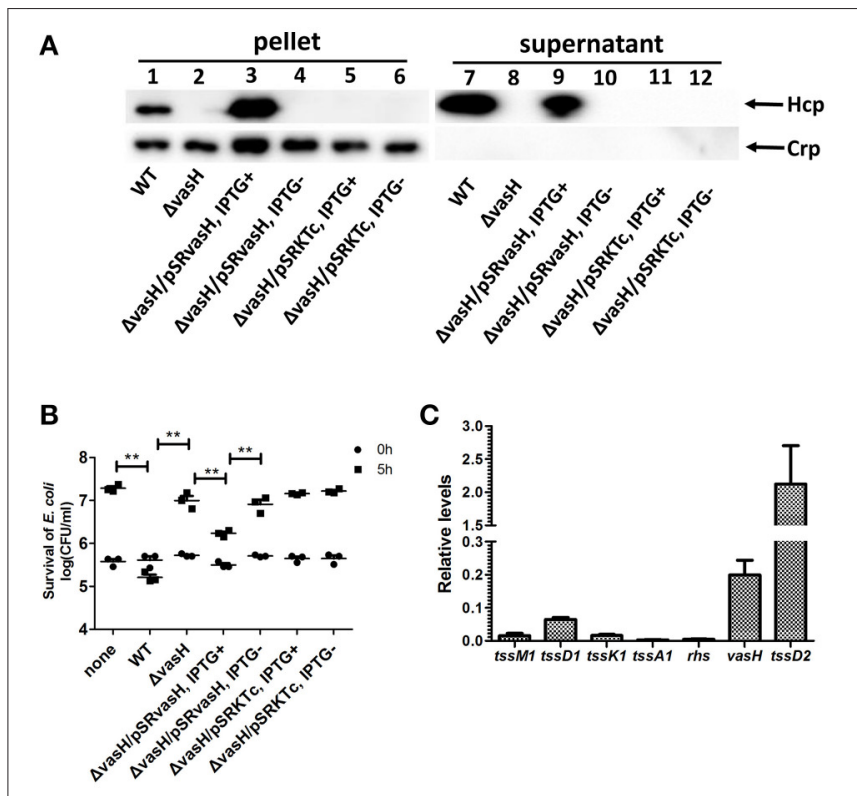

FIGURE 7 | VfIT6SS2-dependent competition between V. fluvialis 85003 and $\boldsymbol{E}$. coli strain MG1655. (A) Immunoblot analysis of Hcp secretion by the vasH mutant and the trans-complemented strains. Lanes 1-6, cell pellets; lanes 7-12, culture supernatants. The arrows indicate the reaction bands of the Hcp and Crp proteins. (B) The survival of the rifampicin-resistant E. coli strain MG1655 was determined after $5 \mathrm{~h}$ co-culture with $V$. fluvialis 85003 (WT), $\Delta$ vasH or the trans-complemented strains at $30^{\circ} \mathrm{C}$ on LB agar plates containing $340 \mathrm{mM} \mathrm{NaCl}$. The data represent three independent experiments. ${ }^{* *}$,Significant differences between sample groups at $5 \mathrm{~h}$ as determined by an unpaired, two-tailed Student's $t$-test $(P<0.001)$. None, medium only; WT, wild type. (C) qRT-PCR analysis of the mRNA abundance of selected VfIT6SS1 and VfIT6SS2 genes. The $V$. fluvialis strain 85003 was grown at $30^{\circ} \mathrm{C}$ in LB containing $340 \mathrm{mM}$ of $\mathrm{NaCl}$. RNA was extracted, and the mRNA abundances of selected VfIT6SS1 genes (tssM1, tss D1, tssK1, tssA1, and $r h s$ ) and VfIT6SS2 genes (vasH and tssD2) were determined by qRT-PCR as described in the Materials and Methods. Each value is the average of three independent cultures. The error bars indicate standard deviations from the mean. 
VflT6SS1 genes located at the beginning of the VflT6SS1 gene cluster ( $r h s, t s s A 1, t s s D 1$ ), and two other genes at its end (tssK1 and tssM1) using qRT-PCR for bacteria cultured under the same conditions as in the killing assay $(340 \mathrm{mM} \mathrm{NaCl}$ at $30^{\circ} \mathrm{C}$ ). We could not detect TssD1 expression in either the cell pellet or the supernatant by a Western blot analysis (data not shown). qRT-PCR analyses revealed that the genes selected from the VflT6SS1 cluster showed extremely low mRNA levels $(0.015,0.063,0.016,0.003$, and 0.004 for $t s s M 1, t s s D 1$, tss $K 1$, $t s s A 1$, and $r h s$, respectively) compared to $v a s H$ and $t s s D 2$ from the VflT6SS2 cluster (0.199 and 2.124 for vasH and tssD2, respectively; Figure 7C). These results suggested that VflT6SS1 is likely inactive, at least under the tested conditions.

Since VflT6SS2 contains three copies of tssD2 (namely $t s s D 2 \_a, t s s D 2 \_b$ and $t s s D 2 \_c$ ), we generated individual deletion mutants and a tssD2_ab double-deletion mutant to pinpoint which copies contribute to the function of VflT6SS2. As shown in Figure 8A, deletion of the individual copies ( $t s s \mathrm{D} 2$ a, $t s s D 2$ b or $\left.t s s D I 2 \_c\right)$ did not affect the expression or secretion of $\mathrm{Hcp}$, while the combined deletion of $t s s D 2 \mathrm{a}$ and $t s s D 2 \mathrm{~b}$ completely abolished the secretion of Hcp. Consistently, each deletion mutant of tssD2_a, tssD2_b or tssDI2_c showed similar interbacterial virulence to the wild type organism, but the double mutant $(\Delta t s s D 2 \mathrm{ab})$ displayed significantly decreased

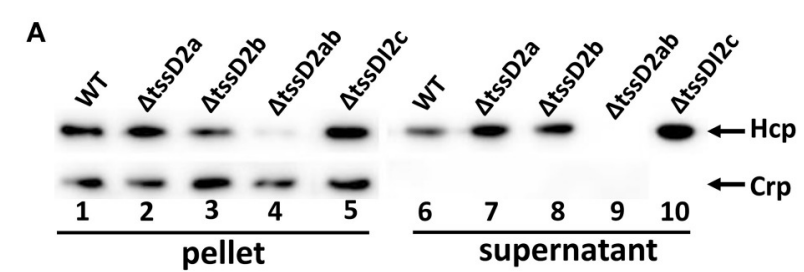

B

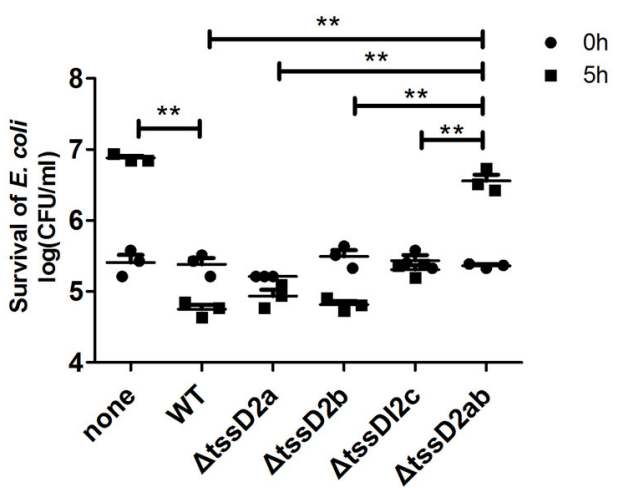

FIGURE 8 | Different contributions of tssD2_a, tssD2_b, and tssD2_c-tss/2_c to the function of VfIT6SS2. (A) Immunoblot analysis of Hcp expression in $\Delta t s s D 2 \mathrm{a}, \Delta t s s D 2 \mathrm{~b}, \Delta t s s D 2 \mathrm{ab}$, and $\Delta t s s D / 2 \mathrm{c}$ cultured at $30^{\circ} \mathrm{C}$. Lanes $1-5$, cell pellets; lanes $6-10$, culture supernatants. The arrows indicate the reaction bands of the Hcp and Crp proteins. (B) The survival of the rifampicin-resistant $E$. coli strain MG1655 was determined after $5 \mathrm{~h}$ of co-culture with $V$. fluvialis $85003(\mathrm{WT}), \Delta t s s D 2 \mathrm{a}, \Delta t s s D 2 \mathrm{~b}, \Delta t s s D 2 \mathrm{ab}$, or $\Delta t s s D / 2 \mathrm{C}$ at $30^{\circ} \mathrm{C}$ on LB agar plates containing $340 \mathrm{mM} \mathrm{NaCl}$. The data represent three independent experiments. ${ }^{*}$,Significant differences between the sample groups at $5 \mathrm{~h}$ as determined by an unpaired, two-tailed Student's t-test $(P<0.05)$. None, medium only. antibacterial virulence (Figure 8B). Based on these results, we speculated that $t s s D 2 \_$a and $t s s D 2$ b function redundantly and are required for VflT6SS2 to mediate its antibacterial activity, while tssD2_c is probably dispensable for the formation of VflT6SS2 structural apparatus or the assembly of functional VflT6SS2.

Taken together, these results demonstrate that VflT6SS2 provides an advantage for $V$. fluvialis in competition with bacterial neighbors, which may contribute to its fitness and pathogenesis.

\section{DISCUSSION}

T6SS is a newly identified protein secretion system in Gramnegative bacteria that functions to antagonize the neighboring cells through the delivery of lethal effector molecules. In this study, we investigated the T6SS machinery in V. fluvialis, an emerging foodborne pathogen. We showed the detailed genetic contents and organization of two T6SS gene clusters in $V$. fluvialis strain 85003 . We found that VflT6SS2 is more widely distributed than VflT6SS1 in different isolates of $V$. fluvialis. VflT6SS2 has three "orphan" hcp-vgrG clusters and has nearly the same genetic organization and gene orientation as the "core" gene cluster of V. cholerae T6SS (VCA0107 VCA0124) except that the homologous counterpart of VCA0122 (vasM) was missing from $V$. fluvialis (Figure 1). The third "orphan" $h c p$-vgrG cluster, tssD2_c-tssI2_c, which closely neighbored three predicted phage integrases, was not found in V. fluvialis 33809, $V$. furnissii NCTC11218 or V. cholerae N16961, implying its unstable characteristics in terms of acquisition and loss.

For the first time, we demonstrated the functional expression of T6SS in V. fluvialis (Figure 2). Previous studies have reported that the expression of T6SS is growth phase-dependent (Ishikawa et al., 2009; Pieper et al., 2009). In this study, we confirmed the growth phase-dependent expression of the T6SS and showed that the growth phase regulates the function of VflT6SS2, i.e., the secretion of the Hcp effector is dependent on the growth phase. As shown in Figure 3, the synthesis of Hcp remains stable throughout the growth phase (from an $\mathrm{OD}_{600}$ of 0.5 to 3.8; Figure $3 \mathrm{~A}$ ), but Hcp secretion was only observed in a narrow range of the growth phase (from an $\mathrm{OD}_{600}$ of 1.0 to 2.0; Figure 3B), suggesting that VflT6SS2 functions only during the late log phase and early stationary phase of the bacterial growth. We know that protein secretion is an energy-consuming process. Turning on the secretion system at certain growth stages may be an adaptive strategy for bacterial cells to avoid unnecessary energy consumption while facilitating cell multiplication and survival. We speculate that this kind of regulation may be achieved through sensing changes in the culture environment, such as the cell density, nutrition consumption, concomitant metabolite accumulation, etc. In support of this hypothesis, it has been reported that quorum sensing, sigma factor RpoN, transcriptional regulators and stress-response-inducing factors activate the T6SS in different bacterial species (Ishikawa et al., 2009; Leung et al., 2011). 
Functional expression of VflT6SS2 was activated under cool $\left(25^{\circ} \mathrm{C}\right)$ and warm $\left(30^{\circ} \mathrm{C}\right)$ temperatures, but was completely repressed at high temperature $\left(37^{\circ} \mathrm{C}\right)$, as evidenced by the lack of Hcp detection in both cell pellets and culture supernatants (Figure 4A) and the extremely low transcriptional level of tssD2 (Figure 4B). The temperature-dependent expression of T6SS was also reported in other species, including Vibrio pathogens such as the $V$. cholerae $\mathrm{O} 1$ serogroup (Ishikawa et al., 2012), $V$. parahaemolyticus (Salomon et al., 2013) and even the zoonotic pathogen, Yersinia pestis (Pieper et al., 2009). The temperaturespecific expression pattern of VflT6SS2, combined with its wide existence in different isolates, suggests that VflT6SS2 probably plays a vital role in $V$. fluvialis bacterial survival and persistence in the environmental niche.

Consistent with what has been reported in $V$. cholerae $\mathrm{O} 1$ strain A1552 (Ishikawa et al., 2012), we also observed that osmolarity induced the secretion of Hcp in $V$. fluvialis under the warm temperature $\left(30^{\circ} \mathrm{C}\right)$ (Figures 5A, 6B). In addition, a higher $\mathrm{NaCl}$ concentration $(>340 \mathrm{mM}$ ) was observed to induce both the expression and secretion of $\mathrm{Hcp}$ in a $\mathrm{NaCl}$ concentration-dependent manner at $37^{\circ} \mathrm{C}$, a temperature which is usually associated with a lack of Hcp. Furthermore, a clear dose-related response was observed (Figure 5C). Similar to what has reported in $V$. cholerae (Ishikawa et al., 2012), qRT-PCR analyses revealed that high salinity promoted the transcription of $t s s \mathrm{D} 2$ and the VflT6SS2 gene cluster (Figures 5B,D), which may be the main reason for the activation of VflT6SS2. However, other possibilities cannot be excluded, such as an increase in Hcp secretion due to increased contraction of VipA/VipB sheath tubules, which may work more rapidly and efficiently in response to various environmental stimuli. This may be supported by the observation that a high salt concentration improves the stability of the VipA/VipB complex in vitro (Bröms et al., 2013). It is also possible that the high salinity affected the dynamic cycle of the sheath tubules (assembly, contraction, disassembly, and reassembly; Basler et al., 2012).

It is worth noting that the range of salinity inducing the secretion and expression of Hcp in $V$. cholerae was much narrower than that in $V$. fluvialis. The presence of $\mathrm{NaCl}$ at 595 $\mathrm{mM}$ completely abolished the secretion of Hcp in $V$. cholerae (Ishikawa et al., 2012), while in V. fluvialis, $855 \mathrm{mM} \mathrm{NaCl}$ still efficiently induced Hcp secretion (Figure 5A). We reasoned that this may be because $V$. fluvialis is a halophilic species and is naturally able to tolerate higher salinity than $V$. cholerae. At the same time, the ability to maintain the functional activity of T6SS at a much higher salinity (higher than $513 \mathrm{mM}$ ) could provide $V$. fluvialis with a competition advantage in the normal marine habitat, where the $\mathrm{NaCl}$ concentration is up to $500 \mathrm{mM}$ (Bröms et al., 2013). V. fluvialis was reported to be the predominant pathogenic Vibrio species isolated from the final effluents of a rural wastewater treatment plant in South Africa (Igbinosa et al., 2009) and could maintain a long-term survival (6 years) in marine sediment (Amel et al., 2008).

Finally, we showed that WT 85003 exhibited antimicrobial activity when co-cultured with E. coli at $30^{\circ} \mathrm{C}$ (Figure 7B). Deletion of vasH compromised the synthesis of Hcp (Figure 7A), and resulted in the loss of bacterial killing activity (Figure 7B).
Complementation of vasH from the pSRvasH plasmid restored the Hcp expression and the bacterial killing activity of the $\Delta$ vasH mutant (Figures 7A,B). qRT-PCR analyses confirmed that under the tested bacterial killing conditions, VflT6SS1 was not functional (Figure 7C). Together, these results demonstrated that the antimicrobial activity is VflT6SS2-mediated and requires the expression of both Hcp and the transcriptional regulator, VasH. Although vasH is necessary for the functional activity of T6SS, we found that it had the lowest detection rate of the various VflT6SS2 genes in the tested isolates. At present, we do not know whether this is due to sequence variation or gene deletion, and will be investigated in subsequent studies. Furthermore, we investigated the contribution of the three copies of $t s s D 2$ to the function and interbacterial virulence of VflT6SS2. Our results showed that the deletion of the individual tssD2 copies did not significantly affect the interbacterial virulence of VflT6SS2, nor did it affect the expression and secretion of Hcp. In contrast, the deletion of both $t s s D 2 \_$a and $t s s D 2$ b abolished the expression of $\mathrm{Hcp}$ and the interbacterial virulence (Figures 8A,B), suggesting that $t s s D 2 \_$a and $t s s D 2 \_b$ function redundantly and are required for VflT6SS2 to mediate its antibacterial activity, while tssDI2_c is probably dispensable, in accordance with its genetic location (i.e., neighbored with phage integrases) and its absence in closelyrelated Vibrio species, such as V. cholerae, V. furnissii, and $V$. fluvialis strain 33809 (Figure 1). The redundancy of function of the two $h c p$ copies (namely tssD2_a and tssD2_b) as structural components and effector proteins of the T6SS was also observed in $V$. cholerae (Ishikawa et al., 2009).

In summary, we obtained evidence that there is at least one functional T6SS in V. fluvialis 85003, and that its expression and the conditions leading to its induction are similar to, but distinct from, those of other Vibrio species. We hypothesize that VflT6SS2 of $V$. fluvialis constitutes an important factor that facilitates successful competition with other organisms in the marine environment. Our findings are helpful to understand the diversity of the distribution, organization, expression, and regulation of T6SS and further broaden the overall knowledge of the pathogenicity and environmental fitness of V. fluvialis.

\section{AUTHORS CONTRIBUTIONS}

$\mathrm{WL}$ and $\mathrm{BK}$ conceived and designed the experiments. $\mathrm{YH}$, $\mathrm{MZ}, \mathrm{WL}, \mathrm{BD}$, and JL performed the experiments. PD and YD performed the bioinformatics analyses. WL, BK, and YH analyzed the data and discussed the results. WL and $\mathrm{YH}$ wrote the paper.

\section{ACKNOWLEDGMENTS}

This work was supported by grants from the National Natural Science Foundation of China (No. 81171640), the project from the Beijing Municipal Science \& Technology Commission (D131100005313016) and the Chinese National Key Basic Research and Development (973) Program (2015CB554201). 


\section{SUPPLEMENTARY MATERIAL}

The Supplementary Material for this article can be found online at: http://journal.frontiersin.org/article/10.3389/fmicb. 2017.00528/full\#supplementary-material

Figure S1 | Growth curves of V. fluvialis strains 85003, VF54, CICC21612, and VF42 incubated in LB media containing $170 \mathrm{mM} \mathrm{NaCl}$ at $30^{\circ} \mathrm{C}$. Error bars indicate the standard deviation of three independent cultures.

\section{REFERENCES}

Ahmed, A. M., Shinoda, S., and Shimamoto, T. (2005). A variant type of Vibrio cholerae SXT element in a multidrug-resistant strain of Vibrio fluvialis. FEMS Microbiol. Lett. 242, 241-247. doi: 10.1016/j.femsle.2004. 11.012

Allton, D. R., Forgione, M. A. Jr., and Gros, S. P. (2006). Cholera-like presentation in Vibrio fluvialis enteritis. South. Med. J. 99, 765-767. doi: 10.1097/01.smj.0000223657.22296.e6

Amel, B. K., Amine, B., and Amina, B. (2008). Survival of Vibrio fluvialis in seawater under starvation conditions. Microbiol. Res. 163, 323-328. doi: 10.1016/j.micres.2006.06.006

Aubert, D. F., Xu, H., Yang, J., Shi, X., Gao, W., Li, L., et al. (2016). A Burkholderia Type VI effector deamidates Rho GTPases to activate the Pyrin Inflammasome and trigger inflammation. Cell Host Microbe 19, 664-674. doi: 10.1016/j.chom.2016.04.004

Basler, M., Pilhofer, M., Henderson, G. P., Jensen, G. J., and Mekalanos, J. J. (2012). Type VI secretion requires a dynamic contractile phage tail-like structure. Nature 483, 182-186. doi: 10.1038/nature10846

Bellet, J., Klein, B., Altieri, M., and Ochsenschlager, D. (1989). Vibrio fluvialis, an unusual pediatric enteric pathogen. Pediatr. Emerg. Care 5, 27-28.

Bhattacharjee, S., Bhattacharjee, S., Bal, B., Pal, R., Niyogi, S. K., and Sarkar, K. (2010). Is Vibrio fluvialis emerging as a pathogen with epidemic potential in coastal region of eastern India following cyclone Aila? J. Health Popul. Nutr. 28, 311-317.

Bingle, L. E., Bailey, C. M., and Pallen, M. J. (2008). Type VI secretion: a beginner's guide. Curr. Opin. Microbiol. 11, 3-8. doi: 10.1016/j.mib.2008.01.006.

Borgeaud, S., Metzger, L. C., Scrignari, T., and Blokesch, M. (2015). The type VI secretion system of Vibrio cholerae fosters horizontal gene transfer. Science 347, 63-67. doi: 10.1126/science.1260064.

Boyer, F., Fichant, G., Berthod, J., Vandenbrouck, Y., and Attree, I. (2009). Dissecting the bacterial type VI secretion system by a genome wide in silico analysis: what can be learned from available microbial genomic resources? BMC Genomics 10:104. doi: 10.1186/1471-2164-10-104

Brenner, D. J., Hickman-Brenner, F. W., Lee, J. V., Steigerwalt, A. G., Fanning, G. R., Hollis, D. G., et al. (1983). Vibrio furnissii (formerly aerogenic biogroup of Vibrio fluvialis), a new species isolated from human feces and the environment. J. Clin. Microbiol. 18, 816-824.

Bröms, J. E., Ishikawa, T., Wai, S. N., and Sjöstedt, A. (2013). A functional VipA-VipB interaction is required for the type VI secretion system activity of Vibrio cholerae O1 strain A1552. BMC Microbiol. 13:96. doi: 10.1186/1471-2180-13-96.

Cabrera Rodríguez, L. E., Monroy, S. P., Morier, L., Ramírez Alvarez, M. M., Fernández Abreu, A., Castro Escarpulli, G., et al. (2005). Severe otitis due to Vibrio fluvialis in a patient with AIDs: first report in the world. Rev. Cubana Med. Trop. 57, 154-155.

Chikahira, M., and Hamada, K. (1988). Enterotoxigenic substance and other toxins produced by Vibrio fluvialis and Vibrio furnissii. Nippon. Juigaku Zasshi 50, 865-873.

Chowdhury, G., Pazhani, G. P., Dutta, D., Guin, S., Dutta, S., Ghosh, S., et al. (2012). Vibrio fluvialis in Patients with Diarrhea, Kolkata, India. Emerg. Infect. Dis. 18, 1868-1871. doi: 10.3201/eid1811.120520

Chowdhury, G., Pazhani, G. P., Nair, G. B., Ghosh, A., and Ramamurthy, T. (2011). Transferable plasmid-mediated quinolone resistance in association with extended-spectrum beta-lactamases and fluoroquinolone-acetylating
Figure S2 | Growth curves of V. fluvialis strain 85003 incubated in LB media containing $170 \mathrm{mM} \mathrm{NaCl}$ at $25^{\circ}, 30^{\circ}$, and $37^{\circ} \mathrm{C}$, respectively. Error bars indicate the standard deviation of three independent cultures.

Figure S3 | Amino acid sequence alignments of the V. fluvialis $85003 \mathrm{VasH}$ protein (VfIVasH) with VasH protein sequences of $\mathrm{V}$. cholerae (VchVasH) and $\boldsymbol{V}$. furnissii (VfuVasH). The identical and highly conserved amino acids are highlighted in black and gray, respectively. The Walker A motif, Walker B motif, the central loop1 region of the ATPase domain and the helix-turn-helix domain are underlined.

aminoglycoside- $6^{\prime}$-N-acetyltransferase in clinical isolates of Vibrio fluvialis. Int J. Antimicrob. Agents 38, 169-173.

Dong, T. G., Ho, B. T., Yoder-Himes, D. R., and Mekalanos, J. J. (2013). Identification of T6SS-dependent effector and immunity proteins by Tnseq in Vibrio cholerae. Proc. Natl. Acad. Sci. U.S.A. 110, 2623-2628. doi: $10.1073 /$ pnas. 1222783110

Han, J. H., Lee, J. H., Choi, Y. H., Park, J. H., Choi, T. J., and Kong, I. S. (2002). Purification, characterization and molecular cloning of Vibrio fluvialis hemolysin. Biochim. Biophys. Acta 1599, 106-114.

Ho, B. T., Dong, T. G., and Mekalanos, J. J. (2014). A view to a kill: the bacterial type VI secretion system. Cell Host Microbe 15, 9-21. doi: 10.1016/j.chom.2013.11.008

Huang, K. C., and Hsu, R. W. (2005). Vibrio fluvialis hemorrhagic cellulitis and cerebritis. Clin. Infect. Dis. 40, e75-e77. doi: 10.1086/429328

Huq, M. I., Alam, A. K., Brenner, D. J., and Morris, G. K. (1980). Isolation of Vibrio-like group, EF-6, from patients with diarrhea. J. Clin. Microbiol. 11, 621-624.

Igbinosa, E. O., Obi, L. C., and Okoh, A. I. (2009). Occurrence of potentially pathogenic vibrios in final effluents of a wastewater treatment facility in a rural community of the Eastern Cape Province of South Africa. Res. Microbiol. 160, 531-537. doi: 10.1016/j.resmic.2009.08.007

Igbinosa, E. O., and Okoh, A. I. (2010). Vibrio fluvialis: an unusual enteric pathogen of increasing public health concern. Int. J. Environ. Res. Public Health 7, 3628-3643. doi: 10.3390/ijerph7103628

Ishikawa, T., Rompikuntal, P. K., Lindmark, B., Milton, D. L., and Wai, S. N. (2009). Quorum sensing regulation of the two hcp alleles in Vibrio cholerae O1 strains. PLoS ONE 4:e6734. doi: 10.1371/journal.pone.0006734

Ishikawa, T., Sabharwal, D., Bröms, J., Milton, D. L., Sjöstedt, A., Uhlin, B. E., et al. (2012). Pathoadaptive conditional regulation of the type VI secretion system in Vibrio cholerae O1 strains. Infect. Immun. 80, 575-584. doi: 10.1128/IAI.05510-11

Khan, S. R., Gaines, J., Roop, R. M. II., and Farrand, S. K. (2008). Broad-host-range expression vectors with tightly regulated promoters and their use to examine the influence of TraR and TraM expression on Ti plasmid quorum sensing. Appl. Environ. Microbiol. 74, 5053-5062. doi: 10.1128/AEM.01098-08

Kitaoka, M., Miyata, S. T., Brooks, T. M., Unterweger, D., and Pukatzki, S. (2011). VasH is a transcriptional regulator of the type VI secretion system functional in endemic and pandemic Vibrio cholerae. J. Bacteriol. 193, 6471-6482. doi: 10.1128/JB.05414-11

Klontz, K. C., and Desenclos, J. C. (1990). Clinical and epidemiological features of sporadic infections with Vibrio fluvialis in Florida, USA. J. Diarrhoeal Dis. Res. 8, 24-26.

Kothary, M. H., Lowman, H., McCardell, B. A., and Tall, B. D. (2003). Purification and characterization of enterotoxigenic El Tor-like hemolysin produced by Vibrio fluvialis. Infect. Immun. 71, 3213-3220.

Lai, C. H., Hwang, C. K., Chin, C., Lin, H. H., Wong, W. W., and Liu, C. Y. (2006) Severe watery diarrhoea and bacteraemia caused by Vibrio fluvialis. J. Infect. 52, e95-e98. doi: 10.1016/j.jinf.2005.05.023

Lee, J. V., Shread, P., Furniss, A. L., and Bryant, T. N. (1981). Taxonomy and description of Vibrio fluvialis sp. nov. (synonym group F. vibrios, group EF6). J. Appl. Bacteriol. 50, 73-94.

Leiman, P. G., Basler, M., Ramagopal, U. A., Bonanno, J. B., Sauder, J. M., Pukatzki, S., et al. (2009). Type VI secretion apparatus and phage tail-associated protein complexes share a common evolutionary origin. Proc. Natl. Acad. Sci. U.S.A. 106, 4154-4159. doi: 10.1073/pnas.0813360106 
Leung, K. Y., Siame, B. A., Snowball, H., and Mok, Y. K. (2011). Type VI secretion regulation: crosstalk and intracellular communication. Curr. Opin. Microbiol. 14, 9-15. doi: 10.1016/j.mib.2010.09.017

Liang, P., Cui, X., Du, X., Kan, B., and Liang, W. (2013). The virulence phenotypes and molecular epidemiological characteristics of Vibrio fluvialis in China. Gut Pathog. 5:6. doi: 10.1186/1757-4749-5-6

Liu, L., Hao, S., Lan, R., Wang, G., Xiao, D., Sun, H., et al. (2015). The Type VI secretion system modulates Flagellar gene expression and secretion in Citrobacter freundii and contributes to adhesion and cytotoxicity to Host Cells. Infect. Immun. 83, 2596-2604. doi: 10.1128/IAI.03071-14

Liu, W. L., Chiu, Y. H., Chao, C. M., Hou, C. C., and Lai, C. C. (2011). Biliary tract infection caused by Vibrio fluvialis in an immunocompromised patient. Infection 39, 495-496. doi: 10.1007/s15010-011-0146-0

Lockwood, D. E., Kreger, A. S., and Richardson, S. H. (1982). Detection of toxins produced by vibrio fluvialis. Infect. Immun. 35, 702-708.

Lu, X., Liang, W., Wang, Y., Xu, J., Zhu, J., and Kan, B. (2014). Identification of genetic bases of Vibrio fluvialis species-specific biochemical pathways and potential virulence factors by comparative genomic analysis. Appl. Environ. Microbiol. 80, 2029-2037. doi: 10.1128/AEM.03588-13

Miyoshi, S., Sonoda, Y., Wakiyama, H., Rahman, M. M., Tomochika, K., Shinoda, S., et al. (2002). An exocellular thermolysin-like metalloprotease produced by Vibrio fluvialis: purification, characterization, and gene cloning. Microb. Pathog. 33, 127-134.

Mougous, J. D., Cuff, M. E., Raunser, S., Shen, A., Zhou, M., Gifford, C. A., et al. (2006). A virulence locus of Pseudomonas aeruginosa encodes a protein secretion apparatus. Science 312, 1526-1530. doi: 10.1126/science.1128393

Pieper, R., Huang, S. T., Robinson, J. M., Clark, D. J., Alami, H., Parmar, P. P., et al. (2009). Temperature and growth phase influence the outer-membrane proteome and the expression of a type VI secretion system in Yersinia pestis. Microbiology 155(Pt 2), 498-512. doi: 10.1099/mic.0.022160-0

Pukatzki, S., Ma, A. T., Sturtevant, D., Krastins, B., Sarracino, D., Nelson, W. C., et al. (2006). Identification of a conserved bacterial protein secretion system in Vibrio cholerae using the Dictyostelium host model system. Proc. Natl. Acad. Sci. U.S.A. 103, 1528-1533. doi: 10.1073/pnas.0510322103

Ratnaraja, N., Blackmore, T., Byrne, J., and Shi, S. (2005). Vibrio fluvialis peritonitis in a patient receiving continuous ambulatory peritoneal dialysis. J. Clin. Microbiol. 43, 514-515. doi: 10.1128/JCM.43.1.514-515.2005

Salomon, D., Gonzalez, H., Updegraff, B. L., and Orth, K. (2013). Vibrio parahaemolyticus type VI secretion system 1 is activated in marine conditions to target bacteria, and is differentially regulated from system 2. PLoS ONE 8:e61086. doi: 10.1371/journal.pone.0061086

Salomon, D., Klimko, J. A., Trudgian, D. C., Kinch, L. N., Grishin, N. V., Mirzaei, H., et al. (2015). Type VI secretion system toxins horizontally shared between marine bacteria. PLoS Pathog. 11:e1005128. doi: 10.1371/journal.ppat.1005128

Schell, M. A., Ulrich, R. L., Ribot, W. J., Brueggemann, E. E., Hines, H. B., Chen, D., et al. (2007). Type VI secretion is a major virulence determinant in Burkholderia mallei. Mol. Microbiol. 64, 1466-1485. doi: 10.1111/j.1365-2958.2007.05734.x

Shalom, G., Shaw, J. G., and Thomas, M. S. (2007). In vivo expression technology identifies a type VI secretion system locus in Burkholderia pseudomallei that is induced upon invasion of macrophages. Microbiology 153(Pt 8), 2689-2699. doi: 10.1099/mic.0.2007/006585-0

Sheng, L., Gu, D., Wang, Q., Liu, Q., and Zhang, Y. (2012). Quorum sensing and alternative sigma factor RpoN regulate type VI secretion system I
(T6SSVA1) in fish pathogen Vibrio alginolyticus. Arch. Microbiol. 194, 379-390. doi: 10.1007/s00203-011-0780-z

Shneider, M. M., Buth, S. A., Ho, B. T., Basler, M., Mekalanos, J. J., and Leiman, P. G. (2013). PAAR-repeat proteins sharpen and diversify the type VI secretion system spike. Nature 500, 350-353. doi: 10.1038/nature12453

Song, L., Huang, Y., Zhao, M., Wang, Z., Wang, S., Sun, H., et al. (2015). A critical role for hemolysin in Vibrio fluvialis-induced IL-1beta secretion mediated by the NLRP3 inflammasome in macrophages. Front. Microbiol. 6:510. doi: 10.3389/fmicb.2015.00510

Srinivasan, V. B., Virk, R. K., Kaundal, A., Chakraborty, R., Datta, B., Ramamurthy, T., et al. (2006). Mechanism of drug resistance in clonally related clinical isolates of Vibrio fluvialis isolated in Kolkata, India. Antimicrob. Agents Chemother. 50, 2428-2432. doi: 10.1128/AAC.01561-05

Suarez, G., Sierra, J. C., Sha, J., Wang, S., Erova, T. E., Fadl, A. A., et al. (2008). Molecular characterization of a functional type VI secretion system from a clinical isolate of Aeromonas hydrophila. Microb. Pathog. 44, 344-361. doi: 10.1016/j.micpath.2007.10.005

Wang, Y., Wang, H., Liang, W., Hay, A. J., Zhong, Z., Kan, B., et al. (2013). Quorum sensing regulatory cascades control Vibrio fluvialis pathogenesis. J. Bacteriol. 195, 3583-3589. doi: 10.1128/JB.00508-13

Weber, B., Hasic, M., Chen, C., Wai, S. N., and Milton, D. L. (2009). Type VI secretion modulates quorum sensing and stress response in Vibrio anguillarum. Environ. Microbiol. 11, 3018-3028. doi: 10.1111/j.1462-2920.2009. 02005.x

Williams, S. G., Varcoe, L. T., Attridge, S. R., and Manning, P. A. (1996). Vibrio cholerae Hcp, a secreted protein coregulated with HlyA. Infect. Immun. 64, 283-289.

Wu, C. F., Lin, J. S., Shaw, G. C., and Lai, E. M. (2012). Acid-induced type VI secretion system is regulated by ExoR-ChvG/ChvI signaling cascade in Agrobacterium tumefaciens. PLoS Pathog. 8:e1002938. doi: 10.1371/journal.ppat.1002938

Wu, R., Zhao, M., Li, J., Gao, H., Kan, B., and Liang, W. (2015). Direct regulation of the natural competence regulator gene tfoX by cyclic AMP (cAMP) and cAMP receptor protein (CRP) in Vibrios. Sci. Rep. 5:14921. doi: 10.1038/srep14921

Yu, Y., Yang, H., Li, J., Zhang, P., Wu, B., Zhu, B., et al. (2012). Putative type VI secretion systems of Vibrio parahaemolyticus contribute to adhesion to cultured cell monolayers. Arch. Microbiol. 194, 827-835. doi: 10.1007/s00203-012-0816-Z

Zheng, J., and Leung, K. Y. (2007). Dissection of a type VI secretion system in Edwardsiella tarda. Mol. Microbiol. 66, 1192-1206. doi: 10.1111/j.1365-2958.2007.05993.x

Conflict of Interest Statement: The authors declare that the research was conducted in the absence of any commercial or financial relationships that could be construed as a potential conflict of interest.

Copyright (c) 2017 Huang, Du, Zhao, Liu, Du, Diao, Li, Kan and Liang. This is an open-access article distributed under the terms of the Creative Commons Attribution License (CC BY). The use, distribution or reproduction in other forums is permitted, provided the original author(s) or licensor are credited and that the original publication in this journal is cited, in accordance with accepted academic practice. No use, distribution or reproduction is permitted which does not comply with these terms. 